J. Inst. Math. Jussieu (2022), 21(6), 1887-1913

doi:10.1017/S1474748021000037 C The Author(s), 2021. Published by Cambridge University 1887 Press. This is an Open Access article, distributed under the terms of the Creative Commons

Attribution licence (http://creativecommons.org/licenses/by/4.0/), which permits unrestricted re-use,

distribution, and reproduction in any medium, provided the original work is properly cited.

\title{
GEOMETRIC LOCAL $\varepsilon$-FACTORS IN HIGHER DIMENSIONS
}

\author{
QUENTIN GUIGNARD \\ Max Planck Institute for Mathematics, Vivatsgasse 7, 53111 Bonn, Germany \\ (guignard@mpim-bonn.mpg.de)
}

(Received 4 May 2020; revised 7 January 2021; accepted 10 January 2021; first published online 2 September 2021)

\begin{abstract}
We prove a product formula for the determinant of the cohomology of an étale sheaf with $\ell$-adic coefficients over an arbitrary proper scheme over a perfect field of positive characteristic $p$ distinct from $\ell$. The local contributions are constructed by iterating vanishing cycle functors as well as certain exact additive functors that can be considered as linearised versions of Artin conductors and local $\varepsilon$-factors. We provide several applications of our higher dimensional product formula, such as twist formulas for global $\varepsilon$-factors.
\end{abstract}

Keywords: étale cohomology, $\varepsilon$-factors, conductors

2020 Mathematics subject classification: $14 \mathrm{~F} 20$

\section{Contents}

1 Introduction $\quad 1888$

2 Preliminaries $r$

3 Nearby and vanishing cycles $\quad 1900$

4 Gabber-Katz extensions and examples of contractions 1902

5 Proof of Theorem $1.2 \quad 1907$

6 Proof of Theorem $1.8 \quad 1910$

$\begin{array}{ll}\text { References } & 1912\end{array}$ 


\section{Introduction}

\section{1.}

Let $k$ be a perfect field of positive characteristic $p$ and let $\bar{k}$ be an algebraic closure of $k$. Let $\Lambda$ be a finite field of characteristic $\ell$ invertible in $k$ or a finite extension of $\mathbb{Q}_{\ell}$. The $\varepsilon$-factor associated with a pair $(X, \mathcal{F})$, where $X$ is a proper $k$-scheme and $\mathcal{F}$ is a bounded constructible complex of étale $\Lambda$-sheaves on $X$, is the Galois line given by

$$
\varepsilon_{\bar{k}}(X, \mathcal{F})=\operatorname{det}\left(R \Gamma\left(X_{\bar{k}}, \mathcal{F}\right)\right)^{-1} .
$$

In this article, we simply consider this object as a homomorphism from $G_{k}=\operatorname{Gal}(\bar{k} / k)$ to $\Lambda^{\times}$. We will also consider the Euler characteristic

$$
\chi(X, \mathcal{F})=\operatorname{rk}\left(R \Gamma\left(X_{\bar{k}}, \mathcal{F}\right)\right) .
$$

In the case where $X$ is a smooth curve over a finite field, it was conjectured by Langlands that the global $\varepsilon$-factor $\varepsilon_{\bar{k}}(X, \mathcal{F})$ should split as a product of local contributions. This conjecture was motivated by the corresponding theory of automorphic local $\varepsilon$-factors. Partial results were obtained by Dwork [2] and Deligne [1], and the product formula for $\ell$-adic sheaves on a smooth curve over a finite field was proved in its full generality by Laumon ([10], Theorem 3.2.1.1). The case of a smooth curve over an arbitrary perfect field of positive characteristic was handled in [6]. An alternative approach by 'spreading out' in order to reduce to the finite field case was also given by Yasuda in $[17,18,16]$.

In the geometric setting of [6], the local $\varepsilon$-factors are realised as determinants of certain Galois modules arising in the cohomology of Gabber-Katz extensions (cf. [6, Def. 9.2]). These Galois modules are thus linearisations of local $\varepsilon$-factors: these are given by additive functors, whose determinants yield the local factors. In this article we show how such linearisations, henceforth labelled linearised Artin conductors, allow splitting higher dimensional global $\varepsilon$-factors into a finite product of local contributions. The resulting higher local $\varepsilon$-factors arise as iteration of vanishing cycle functors and of these linearised Artin conductors. Our main result is the following.

Theorem 1.2. Let $k$ be a perfect field of positive characteristic, and let $X$ be a proper $k$-scheme. Let $\Lambda$ be a finite field whose order has image 1 in $k$. Then there exists a collection

$$
\left(E_{x}\right)_{x \in|X|}
$$

of triangulated functors $E_{x}: D_{c}^{b}\left(X_{(x)}, \Lambda\right) \rightarrow D_{c}^{b}(x, \Lambda)$, indexed by the set of closed points of $X$, where we denoted by $X_{(x)}$ the henselisation of $X$ at a point $x$, such that

(1) for any object $\mathcal{F}$ of $D_{c}^{b}(X, \Lambda)$, we have $E_{x}\left(\mathcal{F}_{\mid X_{(x)}}\right) \simeq 0$ for all but finitely many closed points $x$ of $X$;

(2) for any closed point $x$ of $X$, any object $\mathcal{F}$ of $D_{c}^{b}\left(X_{(x)}, \Lambda\right)$ and any object $\mathcal{G}$ of $D_{c}^{b}(x, \Lambda)$, we have an isomorphism

$$
E_{x}\left(\mathcal{F} \otimes \mathrm{sp}^{-1} \mathcal{G}\right) \simeq E_{x}(\mathcal{F}) \otimes \mathcal{G},
$$


where $\mathrm{sp}: X_{(x)} \rightarrow x$ is the canonical specialisation morphism, and this isomorphism is functorial in $\mathcal{F}$ and $\mathcal{G}$;

(3) for any object $\mathcal{F}$ of $D_{c}^{b}(X, \Lambda)$, we have

$$
\begin{aligned}
& \varepsilon_{\bar{k}}(X, \mathcal{F})=\operatorname{det}\left(\bigoplus_{x \in|X|} \operatorname{Ind}_{G_{x}}^{G_{k}} E_{x}\left(\mathcal{F}_{\mid X_{(x)}}\right)\right), \\
& -\chi(X, \mathcal{F})=\operatorname{rk}\left(\bigoplus_{x \in|X|} \operatorname{Ind}_{G_{x}}^{G_{k}} E_{x}\left(\mathcal{F}_{\mid X_{(x)}}\right)\right),
\end{aligned}
$$

where $G_{k}=\operatorname{Gal}(\bar{k} / k)$ is the Galois group of an algebraic closure of $k$ and $G_{x}$ is the Galois group of $\bar{k} / k(x)$. Here we pick an arbitrary $k$-embedding of $k(x)$ in $\bar{k}$ and we identify $D_{c}^{b}(x, \Lambda)$ with the derived category of bounded constructible complexes of $\Lambda$-modules endowed with a continuous action of $G_{x}$.

Remark 1.3. A similar result holds for $\overline{\mathbb{Q}}_{\ell}$-sheaves, with $\ell$ invertible in $k$, though we restrict throughout this text to the case of finite coefficients to simplify the exposition. The same remark applies to Theorems 1.7, 1.8, 1.9 and 1.10.

Theorem 1.2 will be proved in 5.1 for $X=\mathbb{P}_{k}^{1}$ and in Section 5 in general. It will be clear from the proof that the resulting functors $E_{x}$ are not uniquely determined by the conditions in the conclusion of Theorem 1.2. The factorisation of $\varepsilon_{\bar{k}}(X, \mathcal{F})$ in Theorem 1.2 can be written as

$$
\left.\varepsilon_{\bar{k}}(X, \mathcal{F})=\prod_{x \in|X|} \delta_{x / k}^{\operatorname{rk}\left(E _ { x } \left(\mathcal{F}_{\mid X}(x)\right.\right.}\right) \operatorname{det}\left(E_{x}\left(\mathcal{F}_{\left.\mid X_{(x)}\right)}\right) \circ \operatorname{ver}_{x / k}\right.
$$

where $\delta_{x / k}: G_{k} \rightarrow\{ \pm 1\}$ is the signature homomorphism for the left action of $G_{k}$ on $G_{k} / G_{x}$, and $\operatorname{ver}_{x / k}: G_{k}^{\mathrm{ab}} \rightarrow G_{x}^{\mathrm{ab}}$ is the transfer homomorphism associated with the finite index subgroup $G_{x}$ of $G_{k}$. The factors

$$
\operatorname{det}\left(E_{x}\left(\mathcal{F}_{\mid X_{(x)}}\right)\right)
$$

thus play the role of local $\varepsilon$-factors in higher dimension.

Remark 1.4. It is natural to ask whether it is possible to give a similar product formula determining the line $\operatorname{det}(R \Gamma(X, \mathcal{F}))^{-1}$ up to unique isomorphism, functorially in $\mathcal{F}$, when the base field $k$ is algebraically closed. It will be clear from the proof of Theorem 1.2 that a positive answer to the latter question in the case $X=\mathbb{P}_{k}^{1}$ implies a positive answer for any proper $k$-scheme $X$. However, we do not have any reason to believe that the answer to this question should indeed be positive.

\section{5 .}

In [13], Takeuchi proves a microlocalisation result for the global $\varepsilon$-factor in higher dimension, thereby generalising Saito's microlocal index formula from [11]. Unfortunately, Takeuchi's microlocal formula does not provide a decomposition of the Galois character 
$\varepsilon_{\bar{k}}(X, \mathcal{F})$ but rather of its restriction to $\operatorname{Gal}\left(\bar{k} / k^{\prime}\right)$, where $k^{\prime}$ is a finite extension of $k$ contained in $\bar{k}$, depending on $\mathcal{F}$. If $k$ is a finite field, this is equivalent to describing the determinant of the Frobenius element of $k$ on $R \Gamma\left(X_{\bar{k}}, \mathcal{F}\right)$, modulo roots of unity.

\section{6.}

Theorem 1.2 admits the following immediate consequence.

Theorem 1.7. Let $X$ be a proper $k$-scheme. Let $\mathcal{F}_{1}$ and $\mathcal{F}_{2}$ be objects of $D_{c}^{b}(X, \Lambda)$ such that $\mathcal{F}_{1 \mid X_{(x)}}$ is isomorphic to $\mathcal{F}_{2 \mid X_{(x)}}$ for any closed point $x$ of $X$. Then we have

$$
\begin{aligned}
\chi\left(X, \mathcal{F}_{1}\right) & =\chi\left(X, \mathcal{F}_{2}\right), \\
\varepsilon_{\bar{k}}\left(X, \mathcal{F}_{1}\right) & =\varepsilon_{\bar{k}}\left(X, \mathcal{F}_{2}\right) .
\end{aligned}
$$

The conclusion of 1.7 regarding Euler characteristics is originally due to Deligne, whose proof was written by Illusie in [7]. The part concerning $\varepsilon$-factors appears to be new, though the case of a finite base field alternatively follows from the Grothendieck-Lefschetz trace formula, as was pointed out to the author by Takeshi Saito.

Theorem 1.8. Let $S$ be a Henselian trait of equicharacteristic p, with closed point $s$ such that $k(s)$ is perfect. Let $f: X \rightarrow S$ be a proper morphism and let $\mathcal{F}_{1}$ and $\mathcal{F}_{2}$ be objects of $D_{c}^{b}(X, \Lambda)$ such that $\mathcal{F}_{1 \mid X_{(x)}}$ is isomorphic to $\mathcal{F}_{2 \mid X_{(x)}}$ for any closed point $x$ of the special fibre $X_{s}$. Then we have

$$
\begin{aligned}
a\left(S, R f_{*} \mathcal{F}_{1}\right) & =a\left(S, R f_{*} \mathcal{F}_{2}\right), \\
\varepsilon_{\bar{s}}\left(S, R f_{*} \mathcal{F}_{1}, \omega\right) & =\varepsilon_{\bar{s}}\left(S, R f_{*} \mathcal{F}_{2}, \omega\right)
\end{aligned}
$$

for any nonzero meromorphic 1-form $\omega$ on $S$. Here we denoted by a $(S,-)$ the Artin conductor (cf. [6], Par. 7.2) and by $\varepsilon_{\bar{s}}(S,-, \omega)$ the geometric local $\varepsilon$-factor (cf. [6], Def. 9.2).

This is a consequence of Theorem 6.1, whose proof relies on Theorem 1.2. The first equality also follows from a theorem of Vidal [15].

Theorem 1.9 (Twist Formula). Let $X$ be a proper $k$-scheme and let $\left(E_{x}\right)_{x \in|X|}$ be as in Theorem 1.2. Let $\mathcal{F}$ be an object of $D_{c}^{b}(X, \Lambda)$, and let $\mathcal{G}$ be a $\Lambda$-local system of constant rank $r$ on $X$. We then have

$$
\begin{aligned}
\chi(X, \mathcal{F} \otimes \mathcal{G}) & =r \chi(X, \mathcal{F}), \\
\varepsilon_{\bar{k}}(X, \mathcal{F} \otimes \mathcal{G}) & =\varepsilon_{\bar{k}}(X, \mathcal{F})^{r} \prod_{x \in|X|}\left(\operatorname{det}\left(\mathcal{G}_{\bar{x}}\right) \circ \operatorname{ver}_{x / k}\right)^{\operatorname{rk}\left(E_{x}(\mathcal{F})\right)} .
\end{aligned}
$$

This is an immediate consequence of Theorem 1.2. A similar twist formula was obtained in [14] in the case of a projective smooth scheme over a finite field. Let us set

$$
c c_{X}(\mathcal{F})=-\sum_{x \in|X|} \operatorname{rk}\left(E_{x}(\mathcal{F})\right)[x]
$$

as a 0 -cycle on $X$. Theorem 1.2 yields $\operatorname{deg}\left(c c_{X}(\mathcal{F})\right)=\chi(X, \mathcal{F})$, and the conclusion of Theorem 1.9 can be written as

$$
\varepsilon_{\bar{k}}(X, \mathcal{F} \otimes \mathcal{G})=\varepsilon_{\bar{k}}(X, \mathcal{F})^{r}\left\langle\operatorname{det}(\mathcal{G}),-c c_{X}(\mathcal{F})\right\rangle,
$$


where the pairing $\langle-,-\rangle$ is defined by

$$
\langle\mathcal{L}, c\rangle=\prod_{i}\left(\mathcal{L}_{\overline{x_{i}}} \circ \operatorname{ver}_{x_{i} / k}\right)^{n_{i}},
$$

for any $\Lambda$-local system $\mathcal{L}$ of rank 1 on $X$ and any 0 -cycle $c=\sum_{i} n_{i}\left[x_{i}\right]$. When $k$ is finite and when $X$ is projective smooth, then the main result of [14] and the class field theory of Kato-Saito [8] yield that $c c_{X}(\mathcal{F})$ coincides in the Chow group $C H_{0}(X)$ with the characteristic class defined in ([11], Definition 5.7). When $k$ is arbitrary and $X$ is projective smooth, one can simply assert that the difference between $c c_{X}(\mathcal{F})$ and Saito's characteristic class belong to the kernel of the pairing 1.9.1. As in ([14], Cor. 5.26), this implies that the formation of Saito's characteristic class on projective smooth $k$-schemes commutes with proper pushforward, up to a 0-cycle in the kernel of the pairing 1.9.1. Finally, we notice that Theorem 1.2 actually yields a slightly stronger twist formula:

Theorem 1.10. Let $X$ be a proper $k$-scheme and let $\left(E_{x}\right)_{x \in|X|}$ be as in Theorem 1.2. Let $r$ be an integer. Let $\mathcal{F}_{1}$ and $\mathcal{F}_{2}$ be objects of $D_{c}^{b}(X, \Lambda)$ such that for any closed point $x$ of $X$ we have $\mathcal{F}_{2 \mid X_{(x)}} \simeq \mathcal{F}_{1 \mid X_{(x)}} \otimes \mathcal{G}_{x}$ for some free $\Lambda$-module $\mathcal{G}_{x}$ of rank $r$ endowed with an admissible action of $\operatorname{Gal}(\bar{k} / k(x))$. We then have

$$
\begin{aligned}
\chi\left(X, \mathcal{F}_{2}\right) & =\operatorname{r\chi }\left(X, \mathcal{F}_{1}\right) \\
\varepsilon_{\bar{k}}\left(X, \mathcal{F}_{2}\right) & =\varepsilon_{\bar{k}}\left(X, \mathcal{F}_{1}\right)^{r} \prod_{x \in|X|}\left(\operatorname{det}\left(\mathcal{G}_{x}\right) \circ \operatorname{ver}_{x / k}\right)^{\operatorname{rk}\left(E_{x}(\mathcal{F})\right)} .
\end{aligned}
$$

\subsection{1 .}

Theorem 1.2, as well as its consequences 1.7, 1.8, 1.9, 1.10, can be generalised to (complexes of) étale sheaves twisted by a 2-cocycle on $G_{k}$ (cf. [6], Section 3). We choose to restrict the exposition to nontwisted sheaves for the sake of simplicity and concision. Let us, however, notice that in the twist formula 1.9, we can allow $\mathcal{G}$ to be a twisted local system, and this ultimately yields a more precise conclusion in the discussion following Theorem 1.9 regarding the commutation of the formation of Saito's characteristic class with proper pushforward.

\subsection{2 .}

Let us now describe the organisation of this article. In Section 2 we give a few sorites on Galois equivariant étale sheaves on schemes. We also study transformations of such sheaves afforded by $\Lambda$-linear contractions (cf. 2.12).

In Section 3 we give a brief review of nearby and vanishing cycles functors, as presented in $([5], \mathrm{XIII})$, and we state a few results regarding the composition of these functors with a $\Lambda$-linear contraction.

In Section 4, we review the theory of Gabber-Katz extensions, and we use it to provide three families of $\Lambda$-linear contractions: Katz's cohomological construction of the Swan module from ([9], Sect. 1.6; cf. 4.5), a functor refining geometric class field theory (cf. 4.7) and a linearised Artin conductor (cf. 4.9) that linearises Artin conductors and geometric 
local $\varepsilon$-factors. Only the second and third of these constructions will be used in the proof of our main results, though Katz's construction was inspirational to us.

Finally, we prove the main Theorem 1.2 in Section 5 . We first provide a stronger result in the case of a projective line in 5.1, by using the product formula from [6]. We then prove in 5.2 and 5.3 using Chow's lemma that it is sufficient to prove Theorem 1.2 in the case of a projective space. The latter case is handled in 5.4 by choosing an arbitrary pencil and by applying to the latter the product formula from 5.1, as well as the functoriality properties from Section 3.

\subsection{Conventions and notation}

Throughout this article, we fix distinct prime numbers $p, \ell$. We fix a nontrivial homomorphism $\psi: \mathbb{F}_{p} \rightarrow \overline{\mathbb{F}}_{\ell}^{\times}$, and every finite field $\Lambda$ of characteristic $\ell$ will be assumed to contain the image of $\psi$. We denote by $\mathcal{L}_{\psi}$ the corresponding Artin-Schreier sheaf on $\mathbb{A}_{\mathbb{F}_{p}}^{1}$. For any $\mathbb{F}_{p}$-scheme $X$ and any global function $f$ in $\Gamma\left(X, \mathcal{O}_{X}\right)$, we denote by $\mathcal{L}_{\psi}\{f\}$ the pullback of $\mathcal{L}_{\psi}$ by the morphism $f: X \rightarrow \mathbb{A}_{\mathbb{F}_{p}}^{1}$.

We write 'qcqs' for 'quasi-compact quasi-separated', and for any scheme $S$ we denote by Ét ${ }_{S}$ the category of qcqs étale $S$-schemes and by $S_{\text {ét }}$ the étale topos of $S$, namely, the topos of sheaves of sets on the site Ét $\mathrm{t}_{S}$.

\section{Preliminaries}

In this section, we record various sorites regarding equivariant sheaves and transformations thereof. Throughout this section, we let $s$ be the spectrum of a field and we let $\bar{s}$ be a separable closure of $s$. We denote by $G_{s}$ the Galois group of $k(\bar{s})$ over $k(s)$. In particular, the group $G_{s}$ acts on the right on $\bar{s}$.

\section{1.}

Let $\theta: Q \rightarrow G_{s}$ be a continuous homomorphism of profinite groups and let $Y$ be an $s$-scheme.

Definition 2.2 ([5] XIII 1.1). Let $\Lambda$ be a ring. Let $\mathcal{F}$ be an étale sheaf of sets (respectively of $\Lambda$-modules) on $Y_{\bar{s}}$.

(1) A (left) action of $Q$ compatible with $\theta$ on $\mathcal{F}$ is a collection $(\sigma(q))_{q \in Q}$ of isomorphisms $\sigma(q): \mathcal{F} \rightarrow \theta(q)_{*} \mathcal{F}$, for each $q$ in $Q$, such that

$$
\theta\left(q_{2}\right)_{*} \sigma\left(q_{1}\right) \circ \sigma\left(q_{2}\right)=\sigma\left(q_{1} q_{2}\right),
$$

for any $q_{1}, q_{2}$ in $Q$.

(2) An action of $Q$ compatible with $\theta$ on $\mathcal{F}$ is continuous if for any qcqs étale $Y$-scheme $U$ the resulting left action of $Q$ on $\mathcal{F}\left(U_{\bar{s}}\right)$ is continuous, when the latter is endowed with the discrete topology.

(3) We denote by $\operatorname{Sh}(Y, Q, \theta)$ (respectively $\operatorname{Sh}(Y, Q, \theta, \Lambda)$ ), or simply by $\operatorname{Sh}(Y, Q)$ (respectively $\operatorname{Sh}(Y, Q, \Lambda)$ ) if no confusion can arise from this abuse of notation, the category of étale sheaves (respectively étale sheaves of $\Lambda$-modules) on $Y_{\bar{s}}$ endowed 
with a continuous action of $Q$ compatible with $\theta$. The morphisms in this category are the morphisms of sheaves on $Y_{\bar{s}}$ that commute with the action of $Q$.

If one takes $Y$ to be the base scheme $s$, then the category $\operatorname{Sh}(Y, Q, \theta)$ (respectively $\operatorname{Sh}(Y, Q, \theta, \Lambda))$ is simply the category of sets (respectively of $\Lambda$-modules) endowed with a continuous left action of $Q$, which we also denoted by $Q-\operatorname{Sets}\left(\operatorname{respectively} Q-\operatorname{Mod}_{\Lambda}\right)$.

Remark 2.3. One can alternatively describe the topos $\operatorname{Sh}(Y, Q, \theta)$ (respectively the abelian category $\operatorname{Sh}(Y, Q, \theta, \Lambda)$ ) as the fibre product $\mathrm{B} Q \times{ }_{s_{\text {ét }}} Y_{\text {ét }}$ (respectively as the category of $\Lambda$-modules in the topos $\left.\mathrm{B} Q \times{ }_{s_{\text {ét }}} Y_{\text {ét }}\right)$, where $\mathrm{B} Q$ is the topos of sets endowed with a continuous left action of $Q$. In particular, the abelian category $\operatorname{Sh}(Y, Q, \theta, \Lambda)$ has enough injectives.

Proposition 2.4. The natural functor $\operatorname{Sh}(Y) \rightarrow \operatorname{Sh}\left(Y, G_{s}\right.$, id) is an equivalence of categories.

This is ([5] XIII 1.1.3(ii)). A quasi-inverse to this functor can be described as follows: To any object $\mathcal{F}$ of $\operatorname{Sh}\left(Y, G_{s}\right.$, id $)$ one associates the étale sheaf on $Y$ whose sections on a quasi-compact étale $Y$-scheme $U$ are given by the $G_{s}$-invariants $\mathcal{F}\left(U_{\bar{s}}\right)^{G_{s}}$.

\section{5.}

Let $\Lambda$ be a ring. Let $Y$ be an $s$-scheme and let $\bar{s} \rightarrow s^{\prime} \rightarrow s$ be a factorisation of $\bar{s} \rightarrow s$, where $s^{\prime}$ is a finite extension of $s$. Let us consider a commutative diagram

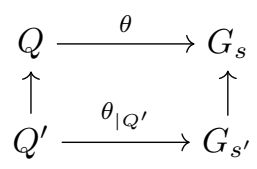

of continuous homomorphisms of profinite groups, with injective vertical arrows. We then have a restriction functor

$$
\operatorname{Res}_{Q^{\prime}}^{Q}: \operatorname{Sh}(Y, Q, \theta, \Lambda) \rightarrow \operatorname{Sh}\left(Y_{s^{\prime}}, Q^{\prime}, \theta_{\mid Q^{\prime}}, \Lambda\right),
$$

obtained by restricting to $Q^{\prime}$ the action of $Q$ on a given object of $\operatorname{Sh}(Y, Q, \Lambda)$. This is an exact and faithful functor. If $Q^{\prime}$ is an open subgroup of $Q$, then the functor $\operatorname{Res}_{Q^{\prime}}^{Q}$ admits a left adjoint, the induction functor

$$
\operatorname{Ind}_{Q^{\prime}}^{Q}: \operatorname{Sh}\left(Y_{s^{\prime}}, Q^{\prime}, \theta_{\mid Q^{\prime}}, \Lambda\right) \rightarrow \operatorname{Sh}(Y, Q, \theta, \Lambda),
$$

which is exact as well.

Example 2.6. Let $U$ be an object of Ét ${ }_{Y_{s^{\prime}}}$. Then

$$
\Lambda_{Q^{\prime}, U}=\operatorname{Ind}_{Q^{\prime}}^{Q} \Lambda\left[\operatorname{Hom}_{Y_{\bar{s}}}\left(-, U \times_{s^{\prime}} \bar{s}\right)\right]
$$

is an object of $\operatorname{Sh}(Y, Q, \Lambda)$. For any object $\mathcal{F}$ of $\operatorname{Sh}(Y, Q, \Lambda)$, the natural homomorphism

$$
\operatorname{Hom}\left(\Lambda_{Q^{\prime}, U}, \mathcal{F}\right) \rightarrow \mathcal{F}\left(U \times_{s^{\prime}} \bar{s}\right)^{Q^{\prime}}
$$

is an isomorphism. 
Proposition 2.7. Let $\mathcal{F}$ be an injective object in $\operatorname{Sh}(Y, Q, \Lambda)$. Let $\mathcal{U}=\left(U_{i} \rightarrow U\right)_{i \in I}$ be a finite cover in $\mathrm{E}_{Y_{\bar{s}}}$. Then for any $j>0$, the Čech cohomology group $H^{j}(\mathcal{U}, \mathcal{F})$ vanishes.

Because $I$ is finite, and because any object of Ét $t_{Y_{\bar{s}}}$ is qcqs, there exists a factorisation $\bar{s} \rightarrow s^{\prime} \rightarrow s$ as in 2.5 and a cover $\mathcal{U}^{\prime}=\left(U_{i}^{\prime} \rightarrow U^{\prime}\right)_{i \in I}$ in Ét $t_{Y_{s^{\prime}}}$ such that $\mathcal{U}$ is isomorphic to $\mathcal{U}^{\prime} \times{ }_{s^{\prime}} \bar{s}=\left(U_{i}^{\prime} \times{ }_{s^{\prime}} \bar{s} \rightarrow U^{\prime} \times{ }_{s^{\prime}} \bar{s}\right)_{i \in I}$. We henceforth assume that $\mathcal{U}$ is actually equal to $\mathcal{U}^{\prime} \times s^{\prime} \bar{s}$.

For any open subgroup $Q^{\prime}$ of $Q$ whose image by $\theta$ is contained in $G_{s^{\prime}}$, let us consider the chain complex

$$
\Lambda_{Q^{\prime}, \mathcal{U}^{\prime}}: \cdots \rightarrow \bigoplus_{i_{0}, i_{1} \in I} \Lambda_{Q^{\prime}, U_{i_{0}}^{\prime} \times_{U^{\prime}} U_{i_{1}}^{\prime}} \rightarrow \bigoplus_{i_{0} \in I} \Lambda_{Q^{\prime}, U_{i_{0}}^{\prime}} \rightarrow 0 \rightarrow \cdots
$$

where the last nonzero term is placed in degree 0 (cf. Example 2.6 for the notation). If $\breve{\mathcal{C}} \bullet(\mathcal{U}, \mathcal{F})$ is the Cech complex of $\mathcal{F}$ with respect to the cover $\mathcal{U}$, then the natural homomorphism

$$
\operatorname{Hom}\left(\Lambda_{Q^{\prime}, \mathcal{U}^{\prime}}, \mathcal{F}\right) \rightarrow \check{\mathcal{C}} \bullet(\mathcal{U}, \mathcal{F})^{Q^{\prime}}
$$

is an isomorphism (cf. Example 2.6). The natural homomorphism $\Lambda_{Q^{\prime}, \mathcal{U}^{\prime}} \rightarrow \Lambda_{Q^{\prime}, U^{\prime}}$ [0] is a quasi-isomorphism by $([12] 03 \mathrm{AT})$ and by exactness of the induction functor $\operatorname{Ind}_{Q^{\prime}}^{Q}$. By applying the exact functor $\operatorname{Hom}(-, \mathcal{F})$, this yields that the natural homomorphism from $\mathcal{F}(U)^{Q^{\prime}}$ to $\check{\mathcal{C}}^{\bullet}(\mathcal{U}, \mathcal{F})^{Q^{\prime}}$ is a quasi-isomorphism. The conclusion then follows by taking the colimit over all open subgroups $Q^{\prime}$ of $Q$ with image by $\theta$ contained in $G_{s^{\prime}}$.

\section{8 .}

Let $\theta: Q \rightarrow G_{s}$ be a continuous homomorphism of profinite groups and let $\Lambda$ be a ring. Let $f: Y^{\prime} \rightarrow Y$ be a quasi-compact morphism of $s$-schemes. For any object $\mathcal{F}$ of $\operatorname{Sh}\left(Y^{\prime}, Q, \theta, \Lambda\right)$, with corresponding action $(\sigma(q))_{q \in Q}$, the data $\left(f_{*} \sigma(q)\right)_{q \in Q}$ are a continous action of $Q$ on $f_{*} \mathcal{F}$ compatible with $\theta$. We thus obtain a functor

$$
f_{*}: \operatorname{Sh}\left(Y^{\prime}, Q, \theta, \Lambda\right) \rightarrow \operatorname{Sh}(Y, Q, \theta, \Lambda) .
$$

We similarly have a functor

$$
f^{-1}: \operatorname{Sh}(Y, Q, \theta, \Lambda) \rightarrow \operatorname{Sh}\left(Y^{\prime}, Q, \theta, \Lambda\right)
$$

which is a left adjoint to $f_{*}$.

Remark 2.9. With the description from Remark 2.3, this pair of adjoint functors is induced by the morphism of toposes id $\times f$ from $\mathrm{B} Q \times_{s_{\text {ét }}} Y_{\text {ét }}^{\prime}$ to $\mathrm{B} Q \times_{s_{\text {ét }}} Y_{\text {ét }}$.

\subsection{0.}

Let $\theta: Q \rightarrow G$ be a surjective continuous homomorphism of profinite groups and let $I$ be its kernel. Let $\Lambda$ be a ring. For any element $q$ of $Q$ and any object $M$ of $I-\operatorname{Mod}_{\Lambda}$, we denote by $q^{*} M$ the object of $I-\operatorname{Mod}_{\Lambda}$ with underlying $\Lambda$-module $M$, on which an element $t$ of $I$ acts as $m \mapsto q t q^{-1} m$. This yields a $\Lambda$-linear functor

$$
q^{*}: I-\operatorname{Mod}_{\Lambda} \rightarrow I-\operatorname{Mod}_{\Lambda},
$$


and we have $\left(q_{1} q_{2}\right)^{*}=q_{2}^{*} q_{1}^{*}$. For any element $q$ of $I$, we have a $\Lambda$-linear natural transformation

$$
\lambda_{q}: \mathrm{id} \rightarrow q^{*}
$$

given on an object $M$ of $I-\operatorname{Mod}_{\Lambda}$ by the homomorphism $m \mapsto q m$.

\subsection{1.}

For any element $g$ of $G$, we can form the diagram $\left(q^{*} M\right)_{q \in \theta^{-1}(g)}$ with transition homomorphisms $q_{1}^{*} M \rightarrow q_{2}^{*} M$ given by $\lambda_{q_{1}^{-1} q_{2}} q_{1}^{*}$. We set

$$
g^{*} M=\operatorname{colim}_{q \in \theta^{-1}(g)} q^{*} M
$$

to be the (filtered) colimit of this diagram. This yields a functor $g^{*}$ from $I-\operatorname{Mod}_{\Lambda}$ to itself. The homomorphisms $\left(\lambda_{q}\right)_{q \in \theta^{-1}(g)}$ define a $\Lambda$-linear natural transformation

$$
\lambda_{g}: \mathrm{id} \rightarrow g^{*} \text {. }
$$

Definition 2.12. A $\Lambda$-linear $(Q, I)$-contraction is a $\Lambda$-linear functor

$$
T: I-\operatorname{Mod}_{\Lambda} \rightarrow \operatorname{Mod}_{\Lambda},
$$

endowed with a collection $\tau=(\tau(g))_{g \in G}$ of $\Lambda$-linear natural transformations

$$
\tau(g): T g^{*} \rightarrow T
$$

satisfying the following conditions:

(1) The functor $T$ is exact and commutes with small filtered colimits.

(2) If an object $M$ of $I-\operatorname{Mod}_{\Lambda}$ is finitely generated as a $\Lambda$-module, then $T(M)$ is a finitely generated $\Lambda$-module, and there exists an open subgroup $Q^{\prime}$ of $Q$ such that for any element $q$ of $Q^{\prime}$ we have $q^{*} M=M$ as an $I$-module and for $g=\theta(q)$, the composition of $\tau(g)_{M}: T\left(g^{*} M\right) \rightarrow T(M)$ with the isomorphism $T(M)=T\left(q^{*} M\right) \cong$ $T\left(g^{*} M\right)$ is the identity endomorphism of $T(M)$.

(3) For any elements $g_{1}, g_{2}$ of $G$, the natural transformation $\tau\left(g_{1}\right) \circ \tau\left(g_{2}\right) g_{1}^{*}$ from $T g_{2}^{*} g_{1}^{*}$ to $T$ coincides with $\tau\left(g_{1} g_{2}\right)$.

We will give nontrivial examples of $\Lambda$-linear $(Q, I)$-contractions in Section 4 (cf. 4.5, 4.7 and 4.9).

\subsection{3.}

Let $\theta: Q \rightarrow G$ be a surjective continuous homomorphism of profinite groups with kernel $I$ and let $\Lambda$ be a ring. Let $(T, \tau)$ be a $\Lambda$-linear $(Q, I)$-contraction (cf. 2.12). Let $G^{\prime}$ be a closed subgroup of $G$ and let $Q^{\prime}$ be its inverse image by $\theta$. Then one can construct a functor

$$
T_{G^{\prime}}: Q^{\prime}-\operatorname{Mod}_{\Lambda} \rightarrow G^{\prime}-\operatorname{Mod}_{\Lambda}
$$

as follows. For any element $g$ of $G^{\prime}$, we have a $\Lambda$-linear natural transformation

$$
\lambda_{g}: \mathrm{id} \rightarrow g^{*}
$$


of endofunctors of $Q^{\prime}-\operatorname{Mod}_{\Lambda}$ (cf. 2.10). We then obtain a $\Lambda$-linear natural transformation

$$
\tau(g) \circ T\left(\lambda_{g}\right): T \phi \rightarrow T \phi,
$$

where $\phi$ is the forgetful functor from $Q^{\prime}-\operatorname{Mod}_{\Lambda}$ to $I-\operatorname{Mod}_{\Lambda}$. Lemma 2.14 then ensures that for any object $M$ of $Q^{\prime}-\operatorname{Mod}_{\Lambda}$, one obtains a continuous left action of $G^{\prime}$ on $T(M)$ by letting an element $g$ of $Q^{\prime}$ act as $\left(\tau(g) \circ T\left(\lambda_{g}\right)\right)_{M}$.

Lemma 2.14. For any elements $g_{1}, g_{2}$ of $G^{\prime}$ we have

$$
\tau\left(g_{1}\right) \circ T\left(\lambda_{g_{1}}\right) \circ \tau\left(g_{2}\right) \circ T\left(\lambda_{g_{2}}\right)=\tau\left(g_{1} g_{2}\right) \circ T\left(\lambda_{g_{1} g_{2}}\right) .
$$

Moreover, for any object $M$ of $Q^{\prime}-\operatorname{Mod}_{\Lambda}$ and element $m$ of $T(M)$ there exists an open subgroup $G^{\prime \prime}$ of $G^{\prime}$ such that $\tau(g) T\left(\lambda_{g}\right) m=m$ for any element $g$ of $G^{\prime \prime}$.

The first assertion follows from the identities

$$
\begin{aligned}
T\left(\lambda_{g_{1}}\right) \circ \tau\left(g_{2}\right) & =\tau\left(g_{2}\right) g_{1}^{*} \circ T\left(g_{2}^{*} \lambda_{g_{1}}\right), \\
\tau\left(g_{1}\right) \circ \tau\left(g_{2}\right) g_{1}^{*} & =\tau\left(g_{1} g_{2}\right), \\
g_{2}^{*} \lambda_{g_{1}} \circ \lambda_{g_{2}} & =\lambda_{g_{1} g_{2}} .
\end{aligned}
$$

The first equality stems from the fact that $\tau\left(g_{2}\right)$ is a natural transformation, the second follows from $2.12(3)$ and the third identity is tautological. For the last assertion of Lemma 2.14, we can assume by 2.12 (1) that $M$ is finitely generated as a $\Lambda$-module, and the conclusion then follows from $2.12(2)$.

\subsection{5.}

Because $T$ and $T_{G}$ are exact functors, we still denote by $T$ and $T_{G}$ the functors

$$
\begin{aligned}
T & : D\left(I-\operatorname{Mod}_{\Lambda}\right) \rightarrow D\left(\operatorname{Mod}_{\Lambda}\right), \\
T_{G} & : D\left(Q-\operatorname{Mod}_{\Lambda}\right) \rightarrow D\left(G-\operatorname{Mod}_{\Lambda}\right)
\end{aligned}
$$

induced by them on the corresponding derived categories.

Proposition 2.16. We have a bifunctorial isomorphism

$$
T\left(M \stackrel{L}{\otimes}{ }_{\Lambda} N\right) \cong T(M) \stackrel{L}{\otimes}{ }_{\Lambda} N
$$

with $M$ in $D^{-}\left(I-\operatorname{Mod}_{\Lambda}\right)$ and $N$ in $D^{-}\left(\operatorname{Mod}_{\Lambda}\right)$. We similarly have a bifunctorial isomorphism

$$
T_{G}(M \stackrel{L}{\otimes} \Lambda \Lambda) \cong T_{G}(M) \stackrel{L}{\otimes_{\Lambda}} N
$$

with $M$ in $D^{-}\left(Q-\operatorname{Mod}_{\Lambda}\right)$ and $N$ in $D^{-}\left(G-\operatorname{Mod}_{\Lambda}\right)$.

We only prove the first assertion, the second one being similar. Let $\mathcal{C}$ be the $\Lambda$-linear category of bounded above chain complexes with terms in the full subcategory of $\operatorname{Mod}_{\Lambda}$ consisting of free $\Lambda$-modules. Because $T$ is $\Lambda$-linear and commutes with small direct sums by $2.12(1)$, we have a functorial isomorphism

$$
T\left(M \otimes_{\Lambda} N\right) \cong T(M) \otimes_{\Lambda} N
$$


with $M$ in $D^{-}\left(I-\operatorname{Mod}_{\Lambda}\right)$ and $N$ in $\mathcal{C}$. This isomorphism factors through the quotient $D^{-}\left(\operatorname{Mod}_{\Lambda}\right)$ of $\mathcal{C}$ because for any homomorphism $\lambda: N_{1} \rightarrow N_{2}$ in $\mathcal{C}$, which is homotopic to 0 , the corresponding homomorphisms

$$
\begin{aligned}
& T\left(M \otimes_{\Lambda} N_{1}\right) \rightarrow T\left(M \otimes_{\Lambda} N_{2}\right), \\
& T(M) \otimes_{\Lambda} N_{1} \rightarrow T(M) \otimes_{\Lambda} N_{2}
\end{aligned}
$$

vanish in $D^{-}\left(\operatorname{Mod}_{\Lambda}\right)$.

Corollary 2.17. Let $M$ be an object of $I-\operatorname{Mod}_{\Lambda}$ that is flat as a $\Lambda$-module. Then $T(M)$ is a flat $\Lambda$-module.

Indeed, for any $\Lambda$-module $N$, the complex $M \stackrel{L}{\otimes_{\Lambda}} N$ is quasi-isomorphic to $M \otimes_{\Lambda} N[0]$ by flatness of $M$; hence, Proposition 2.16 yields an isomorphism

$$
T(M) \stackrel{L}{\otimes_{\Lambda}} N \cong T\left(M \otimes_{\Lambda} N\right)[0]
$$

in $D^{-}\left(\operatorname{Mod}_{\Lambda}\right)$. In particular, the complex $T(M) \stackrel{L}{\otimes_{\Lambda}} N$ is acyclic in nonzero degrees; hence the flatness of $M$.

\subsection{8.}

Let $\theta: Q \rightarrow G_{s}$ be a surjective continuous homomorphism of profinite groups with kernel $I$ and let $\Lambda$ be a ring. Let us consider a $\Lambda$-linear $(Q, I)$-contraction $(T, \tau)$ (cf. 2.12). Let $Y$ be an $s$-scheme.

Definition 2.19. For any object $\mathcal{F}$ of $\operatorname{Sh}(Y, Q, \Lambda)$, we define $T(\mathcal{F})$ to be the étale sheaf of $\Lambda$-modules on $Y$ whose sections on a qcqs étale $Y$-scheme $U$ are given by the $G_{s}$-invariants $T_{G_{s}}\left(\mathcal{F}\left(U_{\bar{s}}\right)\right)^{G_{s}}$ (cf. 2.13).

The mentioned presheaf is indeed a sheaf by left exactness of the functor $M \mapsto$ $T_{G_{s}}(M)^{G_{s}}$ from $Q-\operatorname{Mod}_{\Lambda}$ to the category of $\Lambda$-modules.

Proposition 2.20. We have a bifunctorial isomorphism

$$
\Gamma(U, T(\mathcal{F})) \underset{\sim}{\longrightarrow}(\Gamma(U, \mathcal{F}))
$$

for $U$ in $\operatorname{Et}_{Y_{\bar{S}}}$ and $\mathcal{F}$ in $\operatorname{Sh}(Y, Q, \Lambda)$.

Ler us consider a factorisation $\bar{s} \stackrel{\varphi_{0}}{\longrightarrow} s^{\prime} \rightarrow s$ where $s^{\prime}$ is a finite extension of $s$ and an object $U^{\prime}$ of Ét $t_{Y_{s^{\prime}}}$ such that $U$ is isomorphic to $U^{\prime} \times_{s^{\prime}, \varphi_{0}} \bar{s}$. We henceforth assume that $U$ is equal to $U^{\prime} \times_{s^{\prime}, \varphi_{0}} \bar{s}$. We have a decomposition

$$
U^{\prime} \times{ }_{s} \bar{s}=\coprod_{\bar{s} \stackrel{\varphi}{\longrightarrow} s^{\prime}} U^{\prime} \times_{s^{\prime}, \varphi} \bar{s}
$$

and hence a splitting

$$
\left.\Gamma\left(U_{\bar{s}}^{\prime}, \mathcal{F}\right) \cong \bigoplus_{\bar{s} \stackrel{\varphi}{\longrightarrow} s^{\prime}} \Gamma\left(U^{\prime} \times_{s^{\prime}, \varphi} \bar{s}\right), \mathcal{F}\right)
$$


and, in particular,

$$
T\left(\Gamma\left(U_{\bar{s}}^{\prime}, \mathcal{F}\right)\right) \cong \bigoplus_{\bar{s} \hookrightarrow_{s^{\prime}}} T\left(\Gamma\left(U^{\prime} \times_{s^{\prime}, \varphi} \bar{s}, \mathcal{F}\right)\right) .
$$

Taking into consideration the action of $G_{s}$ on the left-hand side (cf. 2.13), we obtain an isomorphism

$$
T_{G_{s}}\left(\Gamma\left(U_{\bar{s}}^{\prime}, \mathcal{F}\right)\right) \cong \operatorname{Ind}_{G_{s^{\prime}}}^{G_{s}} T_{G_{s^{\prime}}}\left(\Gamma\left(U^{\prime} \times_{s^{\prime}, \varphi_{0}} \bar{s}, \mathcal{F}\right)\right) .
$$

Taking the $G_{s}$-invariants, this yields

$$
\Gamma\left(U^{\prime}, T(\mathcal{F})\right) \cong T_{G_{s^{\prime}}}\left(\Gamma\left(U^{\prime} \times_{s^{\prime}, \varphi_{0}} \bar{s}, \mathcal{F}\right)\right)^{G_{s^{\prime}}}=T_{G_{s^{\prime}}}(\Gamma(U, \mathcal{F}))^{G_{s^{\prime}}} .
$$

If $\bar{s} \rightarrow s^{\prime \prime} \rightarrow s^{\prime}$ is a factorisation of $\varphi_{0}$, where $s^{\prime \prime}$ is a finite extension of $s^{\prime}$, then replacing $s^{\prime}$ and $U^{\prime}$ by $s^{\prime \prime}$ and $U^{\prime} \times{ }_{s^{\prime}} s^{\prime \prime}$ above yields

$$
\Gamma\left(U^{\prime} \times_{s^{\prime}} s^{\prime \prime}, T(\mathcal{F})\right) \cong T_{G_{s^{\prime}}}(\Gamma(U, \mathcal{F}))^{G_{s^{\prime \prime}}} .
$$

By taking the colimit over $s^{\prime \prime}$, we obtain

$$
\Gamma(U, T(\mathcal{F})) \cong T(\Gamma(U, \mathcal{F})) .
$$

Corollary 2.21. For any geometric point $\bar{y}$ of $Y$, we have a natural isomorphism

$$
T(\mathcal{F})_{\bar{y}} \cong T\left(\mathcal{F}_{\bar{y}}\right)
$$

for $\mathcal{F}$ in $\operatorname{Sh}(Y, Q, \Lambda)$. In particular, the functor

$$
T: \operatorname{Sh}(Y, Q, \Lambda) \rightarrow \operatorname{Sh}(Y, \Lambda)
$$

is exact and commutes with small filtered colimits.

Indeed, we can consider $\bar{y}$ as a geometric point of $Y_{\bar{s}}$ and the conclusion follows by taking the (filtered) colimit of the isomorphisms

$$
\Gamma(U, T(\mathcal{F})) \underset{\sim}{\longrightarrow}(\Gamma(U, \mathcal{F}))
$$

from 2.20, where $U$ runs over qcqs étale neighbourhoods of $\bar{y}$ over $Y_{\bar{s}}$.

Corollary 2.22. Let $f: Y^{\prime} \rightarrow Y$ be a morphism of s-schemes. For any object $\mathcal{F}$ of $\operatorname{Sh}(Y, Q, \Lambda)$, the natural homomorphism

$$
f^{-1} T(\mathcal{F}) \rightarrow T\left(f^{-1} \mathcal{F}\right)
$$

with notation as in 2.8, is an isomorphism.

This is an immediate consequence of 2.21 .

Proposition 2.23. Let $\mathcal{F}$ be an injective object in $\operatorname{Sh}(Y, Q, \Lambda)$. Then for any $U$ in $\operatorname{Et}_{Y_{\bar{s}}}$ and any $j>0$ we have $H^{j}(U, T(F))=0$. 
For any étale cover $\mathcal{U}=\left(U_{i} \rightarrow U\right)_{i \in I}$ in Ét $Y_{\bar{s}}$ with $I$ finite, the Čech complex $\check{\mathcal{C}} \bullet(\mathcal{U}, \mathcal{F})$ is acyclic in nonzero degree by Proposition 2.7. By Proposition 2.20 and by exactness of $T$, we obtain that

$$
\check{\mathcal{C}}^{\bullet}(\mathcal{U}, T(\mathcal{F})) \cong T\left(\check{\mathcal{C}}^{\bullet}(\mathcal{U}, \mathcal{F})\right)
$$

is acyclic as well in nonzero degree. The conclusion then follows from ([12] 03F9).

\subsection{4 .}

Let $\theta, \Lambda$ and $(T, \tau)$ be as in 2.18. Let $f: Y^{\prime} \rightarrow Y$ be a qcqs morphism of $s$-schemes. For any object $U$ of Ét $t_{Y}$, the fibre product $U \times_{Y} Y^{\prime}$ is an object of Et $t_{Y^{\prime}}$. Moreover, for any object $\mathcal{F}$ of $\operatorname{Sh}(Y, Q, \Lambda)$, we have, with the notation from 2.8,

$$
\begin{aligned}
\Gamma\left(U, T\left(f_{*} \mathcal{F}\right)\right) & =T_{G_{s}}\left(f_{*} \mathcal{F}\left(U_{\bar{s}}\right)\right)^{G_{s}} \\
& =T_{G_{s}}\left(\mathcal{F}\left(U_{\bar{s}} \times_{Y_{\bar{s}}} Y_{\bar{s}}^{\prime}\right)\right)^{G_{s}} \\
& =\Gamma\left(U_{*} T(\mathcal{F})\right),
\end{aligned}
$$

and hence a commutative diagram

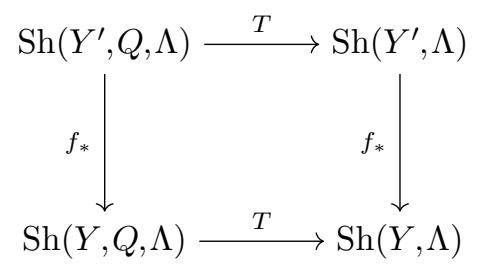

of abelian categories.

Proposition 2.25. We have a natural isomorphism

$$
R f_{*} \circ T \cong T \circ R f_{*}
$$

of functors from $D^{+}\left(Y^{\prime}, Q, \Lambda\right)$ to $D^{+}(Y, \Lambda)$.

Indeed, for any injective object $\mathcal{F}$ of $\operatorname{Sh}\left(Y^{\prime}, Q, \Lambda\right)$, the object $f_{*} \mathcal{F}$ of $\operatorname{Sh}(Y, Q, \Lambda)$ is $T$ acyclic by exactness of $T$ (cf. 2.21). Moreover, for any integer $j>0$ the restriction of $R^{j} f_{*} T(\mathcal{F})$ to $Y_{\bar{s}}$ is the sheaf associated with the presheaf

$$
U \mapsto H^{j}\left(U \times_{Y_{\bar{s}}} Y_{\bar{s}}^{\prime}, T(\mathcal{F})\right),
$$

which vanishes by 2.23 . Thus $T(\mathcal{F})$ is $f_{*}$-acyclic. This implies that the canonical transformations

$$
\begin{aligned}
& R\left(f_{*} \circ T\right) \rightarrow R f_{*} \circ T, \\
& R\left(T \circ f_{*}\right) \rightarrow T \circ R f_{*}
\end{aligned}
$$

are isomorphisms, and the conclusion then follows from the equality $f_{*} \circ T=T \circ f_{*}$. 


\subsection{6 .}

Let $\theta: Q \rightarrow G_{s}$ be a surjective continuous homomorphism of profinite groups with kernel $I$ and let $\Lambda$ be a Noetherian ring. Let $Y$ be a locally Noetherian $s$-scheme. We denote by $\operatorname{Sh}_{c}(Y, Q, \Lambda)$ the full subcategory of $\operatorname{Sh}(Y, Q, \Lambda)$ consisting of objects $\mathcal{F}$ whose underlying sheaf of $\Lambda$-module on $Y_{\bar{s}}$ is constructible (cf. [3], IX 2.3).

Proposition 2.27. Let $(T, \tau)$ be a $\Lambda$-linear $(Q, I)$-contraction and let $\mathcal{F}$ be an object of $\operatorname{Sh}(Y, Q, \Lambda)$.

(1) If the object of $\operatorname{Sh}\left(Y_{\bar{s}}, \Lambda\right)$ underlying $\mathcal{F}$ is locally constant constructible, then $T(\mathcal{F})$ is locally constant constructible.

(2) If the object of $\operatorname{Sh}\left(Y_{\bar{s}}, \Lambda\right)$ underlying $\mathcal{F}$ is constructible, then $T(\mathcal{F})$ is constructible.

Part (1) follows from Corollary 2.21, from 2.12 (2) and from ([3], IX 2.13 (i)). Part (2) follows from (1) and from Corollary 2.22.

\section{Nearby and vanishing cycles}

Let $S$ be a scheme and let $s$ be a point of $S$ such that the local ring $\mathcal{O}_{S, s}$ is a discrete valuation ring. We denote by $\bar{s}$ a separable closure of $s$, by $\eta$ the generic point of the henselisation $S_{(s)}$ of $S$ at $s$ and by $\bar{\eta}$ a separable closure of the generic point $\eta_{\bar{s}}$ of the strict henselisation $S_{(\bar{s})}$.

\section{1.}

Let us denote by $G_{\eta}$ and $G_{s}$ the Galois group of the extensions $\bar{\eta} \rightarrow \eta$ and $\bar{s} \rightarrow$ $s$ respectively. We have a natural specialisation homomorphism $\theta: G_{\eta} \rightarrow G_{s}$. This homomorphism is surjective and we denote by $I_{\eta}$ its kernel, so that we have an exact sequence

$$
1 \rightarrow I_{\eta} \rightarrow G_{\eta} \rightarrow G_{s} \rightarrow 1
$$

of profinite groups. In this section, we apply the definitions and results of Section 2 to $Q=G_{\eta}$ and $\theta$ as above.

\section{2 .}

Let $f: X \rightarrow S$ be an $S$-scheme. Let us consider the commutative diagram

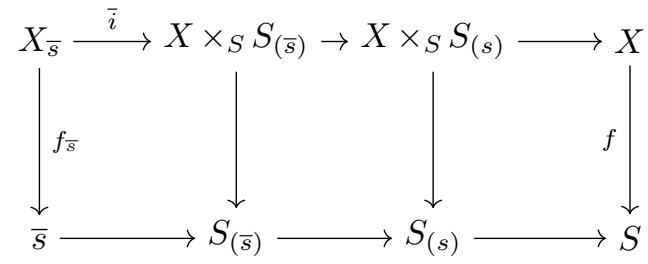

of $S$-schemes, where all squares are Cartesian. 
Definition 3.3 ([5], XIII 1.3.2). Let $\Lambda$ be a ring and let $\mathcal{F}$ be an object of $\operatorname{Sh}(X, \Lambda)$. We denote by $\Psi_{f}^{s}(\mathcal{F})$ the object of $\operatorname{Sh}\left(X_{s}, G_{\eta}, \Lambda\right.$ ) (cf. 2.2) obtained as the pullback by $\bar{i}$ of the sheaf of $\Lambda$-modules on $X \times_{S} S_{(\bar{s})}$ whose sections on an object $U$ of Ét $\operatorname{X}_{X{ }_{S} S_{(\bar{s})}}$ are given by $\Gamma\left(U \times_{S_{(\bar{s})}} \bar{\eta}, \mathcal{F}\right)$.

This defines a left exact functor

$$
\Psi_{f}^{s}: \operatorname{Sh}(X, \Lambda) \rightarrow \operatorname{Sh}\left(X_{s}, G_{\eta}, \Lambda\right)
$$

thereby inducing a right derived functor

$$
R \Psi_{f}^{s}: D^{+}(X, \Lambda) \rightarrow D^{+}\left(X_{s}, G_{\eta}, \Lambda\right),
$$

namely, the nearby cycles functor (cf. [5], XIII 2.1.1).

\section{4.}

Let $i: X_{s} \rightarrow X$ be the natural closed immersion. The natural cospecialisation homomorphism

$$
i^{-1} \rightarrow \Psi_{f}^{s}
$$

yields a triangulated functor from $D^{+}(X, \Lambda)$ to the derived category of the arrow category of $\operatorname{Sh}\left(X_{s}, G_{\eta}, \Lambda\right)$. Its composition with the cone functor is the vanishing cycles functor, which we denote by

$$
R \Phi_{f}^{s}: D^{+}(X, \Lambda) \rightarrow D^{+}\left(X_{s}, G_{\eta}, \Lambda\right) .
$$

We thus have a functorial distinguished triangle

$$
i^{-1} \mathcal{F} \rightarrow R \Psi_{f}^{s} \mathcal{F} \rightarrow R \Phi_{f}^{s}(\mathcal{F}) \stackrel{[1]}{\longrightarrow},
$$

for $\mathcal{F}$ in $D^{+}(X, \Lambda)$.

\section{5.}

Let $\Lambda$ be a Noetherian ring annihilated by an integer invertible on $s$. Let $(T, \tau)$ be a $\Lambda$-linear $\left(G_{\eta}, I_{\eta}\right)$-contraction (cf. 2.12). The composition of the functor $T$ from 2.19 with the vanishing cycles functor from 3.4 yields a triangulated functor

$$
T R \Phi_{f}^{s}: D^{+}(X, \Lambda) \rightarrow D^{+}\left(X_{s}, \Lambda\right) .
$$

We now list a few of its properties.

Proposition 3.6. Let $\mathcal{F}$ be an object of $D_{c}^{b}(X, \Lambda)$. If $f$ is locally of finite type, then $T R \Phi_{f}^{s}$ belongs to $D_{c}^{b}\left(X_{s}, \Lambda\right)$.

Under these assumptions, it follows from ([4], [Th. finitude] Th. 3.2) that $R \Psi_{f}^{s}(\mathcal{F})$ belongs to $D_{c}^{b}\left(X_{s}, \Lambda\right)$; hence, $R \Phi_{f}^{s}(\mathcal{F})$ belongs as well to $D_{c}^{b}\left(X_{s}, \Lambda\right)$ and so does $T R \Phi_{f}^{s}(\mathcal{F})$ by $2.27(2)$. 
Proposition 3.7. Let $h: X^{\prime} \rightarrow X$ be a smooth morphism of $S$-schemes and let $\mathcal{F}$ be an object of $D^{+}(X, \Lambda)$. Then the natural homomorphism

$$
h_{s}^{-1} \operatorname{TR} \Phi_{f}^{s}(\mathcal{F}) \rightarrow T R \Phi_{f h}^{s}\left(h^{-1} \mathcal{F}\right)
$$

is an isomorphism, where $h_{s}: X_{s}^{\prime} \rightarrow X_{s}$ is the morphism induced by $h$. In particular, if $f$ is smooth, then $\operatorname{TR} \Phi_{f}^{s}(\Lambda)$ vanishes.

This follows from the corresponding property of $R \Psi_{f}^{s}$ (cf. [5], XIII 2.1.7.1) and from Corollary 2.22.

Proposition 3.8. Let $h: X^{\prime} \rightarrow X$ be a proper morphism of $S$-schemes and let $\mathcal{F}$ be an object of $D^{+}\left(X^{\prime}, \Lambda\right)$. Then we have a natural homomorphism

$$
R h_{s *} T R \Phi_{f h}^{s}\left(\mathcal{F}^{\prime}\right) \rightarrow T R \Phi_{f}^{s}\left(R h_{*} \mathcal{F}\right)
$$

in $D^{+}\left(X_{s}, \Lambda\right)$. In particular, if $f$ is proper then we have a functorial isomorphism

$$
R \Gamma\left(X_{\bar{s}}, T R \Phi_{f}^{s}(\mathcal{F})\right) \rightarrow T R \Phi_{\text {id }}^{s}\left(R f_{*} \mathcal{F}\right)
$$

in $D^{+}\left(G_{s}-\operatorname{Mod}_{\Lambda}\right)$.

This follows from the corresponding property of $R \Psi_{f}^{s}$ (cf. [5], XIII 2.1.7.2) and from Proposition 2.25 because $h$ is qcqs.

\section{Gabber-Katz extensions and examples of contractions}

In this section, we provide examples of linear contractions (cf. 2.12) whose constructions are based on the theory of Gabber-Katz extensions from [9]. We review the latter in 4.1 by placing these extensions in what we believe to be their rightful context, namely, as pullbacks along a morphism of toposes, the Gabber-Katz retraction (cf. 4.4). Throughout this section, we fix a perfect field $k$ of characteristic $p>0$.

\section{1.}

Let $S$ be a smooth $k$-scheme purely of dimension 1, and let $s$ be a closed point of $S$. Thus, the henselisation $S_{(s)}$ of $S$ at $s$ is a Henselian trait whose residue field $k(s)$ is a finite extension of $k$. Let $\pi$ be a uniformiser of $S_{(s)}$, thereby inducing a morphism

$$
\pi: S_{(s)} \rightarrow \mathbb{A}_{s}^{1}
$$

corresponding to the unique homomorphism $k(s)[t] \rightarrow \mathcal{O}_{S_{(s)}}$ of $k(s)$-algebras sending $t$ to $\pi$. Let us denote by

$$
\pi_{\diamond}: \mathrm{Et}_{S_{(s)}} \rightarrow \mathrm{E}^{\prime} \mathrm{A}_{s}^{1}
$$

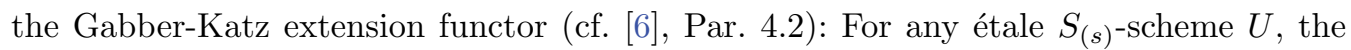
étale $\mathbb{A}_{s}^{1}$-scheme $\pi_{\diamond} U$ is the unique (up to unique isomorphism) such $\mathbb{A}_{s}^{1}$-scheme endowed with an isomorphism from its pullback by $\pi$ to $U$, whose restriction along the open immersion $\mathbb{G}_{m, s} \rightarrow \mathbb{A}_{s}^{1}$ is a special finite étale $\mathbb{G}_{m, s}$-scheme; namely, it is tamely ramified above $\infty$ and its geometric monodromy group has a unique $p$-Sylow (cf. [6], Par. 4.3). 
We will also label special an étale $\mathbb{A}_{s}^{1}$-scheme whose restriction along the open immersion $\mathbb{G}_{m, s} \rightarrow \mathbb{A}_{s}^{1}$ is special or, equivalently, an object of the essential image of $\pi_{\diamond}$.

Proposition 4.2. The functor $\pi_{\diamond}$ has the following properties:

(1) It sends a final object of $\mathrm{É}_{S_{(s)}}$ to a final object of $\mathrm{E}_{\mathbb{A}_{s}^{1}}$.

(2) For any cover $\left(U_{i} \rightarrow U\right)_{i \in I}$ of the site $\mathrm{Ét}_{S_{(s)}}$, the family $\left(\pi_{\diamond} U_{i} \rightarrow \pi_{\diamond} U\right)_{i \in I}$ is a cover in site $\mathrm{Et}_{\mathbb{A}_{s}^{1}}$.

(3) For any diagram $U \rightarrow W \leftarrow V$ in Ét $_{S_{(s)}}$, the natural $\mathbb{A}_{s}^{1}$-morphism

$$
\pi_{\diamond}\left(U \times_{W} V\right) \rightarrow \pi_{\diamond}(U) \times_{\pi_{\diamond}(W)} \pi_{\diamond}(V)
$$

is an isomorphism.

The $\mathbb{A}_{s}^{1}$-scheme $\mathbb{A}_{s}^{1}$ is special and its pullback by $\pi$ is $S_{(s)}$; hence, $\pi_{\diamond} S_{(s)}$ isomorphic to $\mathbb{A}_{s}^{1}$. This proves (1). In order to prove (2), we can assume that $U$ is connected, because the functor $\pi_{\diamond}$ commutes with finite disjoint unions. Moreover, we have that $\pi^{-1} \pi_{\diamond} U_{i}$ is isomorphic to $U_{i}$, so that $\left(\pi^{-1} \pi_{\diamond} U_{i}\right)_{i \in I}$ covers $\pi^{-1} \pi_{\diamond} U$; hence it is sufficient to prove that $\left(j^{-1} \pi_{\diamond} U_{i}\right)_{i \in I}$ covers $j^{-1} \pi_{\diamond} U$, where $j: \mathbb{G}_{m, s} \rightarrow \mathbb{A}_{s}^{1}$ is the natural open immersion. Let $i_{0}$ in $I$ be such that the image of $U_{i_{0}}$ in $U$ contains the generic point of $U$. Then the image of $j^{-1} \pi_{\diamond} U_{i_{0}}$ in $j^{-1} \pi_{\diamond} U$ is nonempty. Because the morphism $j^{-1} \pi_{\diamond} U_{i_{0}} \rightarrow j^{-1} \pi_{\diamond} U$ is finite étale, its image is closed and open in the connected scheme $j^{-1} \pi_{\diamond} U$. Consequently, the morphism $j^{-1} \pi_{\diamond} U_{i_{0}} \rightarrow j^{-1} \pi_{\diamond} U$ is surjective. Let us now prove (3). Let

$$
\alpha: \pi_{\diamond}\left(U \times_{W} V\right) \rightarrow \pi_{\diamond}(U) \times_{\pi_{\diamond}(W)} \pi_{\diamond}(V)
$$

be the morphism under consideration. Then $\pi^{-1} \alpha$ is an isomorphism, and we infer from the characterisation of $\pi_{\diamond}$ (cf. 4.1) that (3) holds if and only if $X=\pi_{\diamond}(U) \times_{\pi_{\diamond}(W)} \pi_{\diamond}(V)$ is a special $\mathbb{A}_{s}^{1}$-scheme. Because $\pi_{\diamond}(U)$ and $\pi_{\diamond}(V)$ are tamely ramified above infinity, so is $X$. Moreover, the geometric monodromy group of $j^{-1} X \rightarrow \mathbb{G}_{m, s}$ is a subgroup of the product of those of $j^{-1} \pi_{\diamond}(U)$ and $j^{-1} \pi_{\diamond}(V)$, and the class of finite groups that admit a unique $p$-Sylow is stable by finite products and subobjects; hence, the geometric monodromy group of $j^{-1} X \rightarrow \mathbb{G}_{m, s}$ belongs to that class. Thus, $X$ is a special $\mathbb{A}_{s}^{1}$-scheme, and (3) holds.

Corollary 4.3. The functor $\pi_{\diamond}: \mathrm{E}_{S_{(s)}} \rightarrow \mathrm{E}_{\mathbb{A}_{s}^{1}}$ is continuous and the corresponding

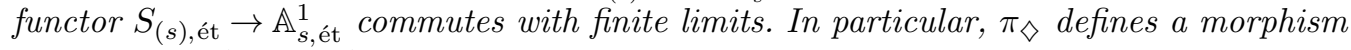
of sites from $\mathrm{Et}_{\mathbb{A}_{s}^{1}}$ to $\mathrm{Et}_{S_{(s)}}$.

This follows from Proposition 4.2 and from the fact that Ét ${ }_{S_{(s)}}$ is an essentially small category.

Definition 4.4. Let $(S, s, \pi)$ be as in 4.1. The Gabber-Katz retraction associated with

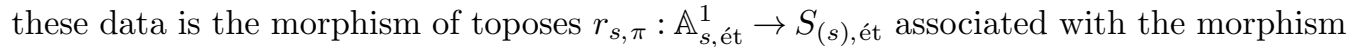
of sites $\pi_{\diamond}$ (cf. 4.3).

The composition of $r_{s, \pi}$ with the morphism $\pi: S_{(s) \text {, ét }} \rightarrow \mathbb{A}_{s \text {, ét }}^{1}$ of toposes induced by $\pi$ is (equivalent to) the identity morphism of $S_{(s)}$, ét ; hence the terminology 'retraction'. 
The pair $r_{s, \pi}=\left(r_{s, \pi}^{-1}, r_{s, \pi *}\right)$ of adjoint functors can be described as follows. For an object $\mathcal{F}$ of $\mathbb{A}_{s \text {,ét }}^{1}$, the object $r_{s, \pi *} \mathcal{F}$ is the sheaf on $\operatorname{Et}_{S_{(s)}}$ given by $U \mapsto \mathcal{F}\left(\pi_{\diamond} U\right)$. Moreover, the functor $r_{s, \pi}^{-1}: S_{(s) \text {,ét }} \rightarrow \mathbb{A}_{s \text {, ét }}^{1}$ is the Gabber-Katz extension functor, sending a representable sheaf $\underline{U}$ to the representable sheaf $\pi_{\diamond} U$.

\section{5.}

Let $\Lambda$ be a finite field of characteristic $\ell$, and let $(S, s, \pi)$ be as in 4.1. Let $\eta_{s}$ be the generic point of $S_{(s)}$, let $\bar{s}$ be a separable closure of $s$ and let $\bar{\eta}_{s}$ be a separable closure of $\eta_{\bar{s}}$. Let $G_{\eta_{s}}$ and $G_{s}$ be the Galois group of the extensions $\bar{s} \rightarrow s$ and $\bar{\eta}_{s} \rightarrow \eta_{s}$. Let $I_{\eta_{s}}$ be the kernel of the surjective specialisation homomorphism $G_{\eta_{s}} \rightarrow G_{s}$. For an object $M$ of $G_{\eta_{s}}-\operatorname{Mod}_{\Lambda}$, we denote by $M_{\text {! }}$ the extension by zero to $S_{(s)}$ of the corresponding sheaf on $\eta_{s}$. In ([9], Sect. 1.6), Katz considered the functor defined by

$$
\operatorname{Sw}(M)=H^{1}\left(\mathbb{A} \frac{1}{s}, r_{\bar{s}, \pi}^{-1} M_{!}\right)
$$

for $M$ in $I_{\eta_{s}}-\operatorname{Mod}_{\Lambda}$, where $r_{\bar{s}, \pi}$ is the canonical retraction from 4.4.

For an element $g$ of $G_{s}$, let us construct a natural transformation

$$
\tau(g): \mathrm{Sw} g^{*} \rightarrow \mathrm{Sw}
$$

on $I_{\eta_{s}}-\operatorname{Mod}_{\Lambda}$ (cf. 2.11). We have a functorial isomorphism $\left(g^{*} M\right) ! \simeq g^{*} M_{!}$, where $g$ acts naturally on $S_{(\bar{s})}$. We then have functorial isomorphisms

$$
\begin{aligned}
\operatorname{Sw}\left(g^{*} M\right) & \simeq H^{1}\left(\mathbb{A}_{\frac{s}{s}}, r_{\bar{s}, \pi}^{-1} g^{*} M_{!}\right) \\
& \simeq H^{1}\left(\mathbb{A}_{\bar{s}}, g^{*} r_{\bar{s}, \pi}^{-1} M_{!}\right),
\end{aligned}
$$

where $g$ acts naturally on $\mathbb{A} \frac{1}{s}$. We now compose with the natural homomorphism from $g^{*} r_{\bar{s}, \pi}^{-1} M_{\text {! }}$ to $\left(g^{-1}\right)_{*} r_{\bar{s}, \pi}^{-1} M_{!}$, in order to obtain a functorial homomorphism from $\operatorname{Sw}\left(g^{*} M\right)$ to

$$
H^{1}\left(\mathbb{A} \frac{1}{s},\left(g^{-1}\right)_{*} r_{\bar{s}, \pi}^{-1} M_{!}\right)=\operatorname{Sw}(M) .
$$

Proposition 4.6. The couple $\left(\mathrm{Sw},(\tau(g))_{g \in G_{s}}\right)$ is a $\left(G_{\eta_{s}}, I_{\eta_{s}}\right)$-contraction. Moreover, we have

$$
\operatorname{rk}(\operatorname{Sw}(M))=\operatorname{sw}(M),
$$

where $\operatorname{sw}(M)$ is the Swan conductor of $M$.

For the exactness of Sw see ([9], Par. 1.6). Moreover, the functor Sw commutes with small filtered colimits because that property is satisfied by the functors $(-) !, r_{\bar{s}, \pi}^{-1}$ and $H^{1}\left(\mathbb{A} \frac{1}{s},-\right)$. Thus, 2.12(1) is satisfied. The composition formula 2.12(3) is immediate, whereas 2.12(2) follows from preservation of constructibility in étale cohomology.

4.7.

We retain the notation from 4.5. Let us set $\tilde{\pi}=\left(1-\pi^{-1}\right)^{-1}$. The uniformiser $\pi$ is uniquely determined by $\tilde{\pi}$, because we have $\pi=\left(1-\tilde{\pi}^{-1}\right)^{-1}$. Let $\overline{1}: \bar{s} \rightarrow \mathbb{A} \frac{1}{s}$ be the $\bar{s}$-point 1 of $\mathbb{A} \frac{1}{s}$. 
Let us set

$$
\operatorname{CFT}_{\tilde{\pi}}(M)=r_{\bar{s}, \pi}^{-1}\left(M_{!}\right)_{\overline{1}},
$$

for $M$ in $I_{\eta_{s}}-\operatorname{Mod}_{\Lambda}$. This is naturally a $\Lambda$-linear $\left(G_{\eta_{s}}, I_{\eta_{s}}\right)$-contraction, with $\tau(g)$ given for $g$ in $G_{s}$ by the natural composition

$$
\mathrm{CFT}_{\tilde{\pi}}\left(g^{*} M\right)=r_{\bar{s}, \pi}^{-1}\left(g^{*} M_{!}\right)_{\overline{1}} \simeq g^{*} r_{\bar{s}, \pi}^{-1}\left(M_{!}\right)_{\overline{1}} \simeq r_{\bar{s}, \pi}^{-1}\left(M_{!}\right)_{\overline{1}}=\operatorname{CFT}_{\tilde{\pi}}(M) .
$$

The functor $\mathrm{CFT}_{\tilde{\pi}}$ can be considered as constituting a linearised class field theory for $\eta_{s}$, as indicated by the following result.

Proposition 4.8. Let $\Lambda$ be a field of characteristic $\ell$. Let $M$ be an object of $G_{\eta_{s}}-$ $\operatorname{Mod}_{\Lambda}$ and let $D$ be a divisor on $S_{(s)}$ such that $\operatorname{det}(M)$ has ramification bounded $D$ (cf. [6], Def. 5.17). Let $\chi_{\operatorname{det}(M)}$ be the multiplicative local system on $\operatorname{Pic}\left(S_{(s)}, D\right)_{s}$ (cf. [6], Def. 5.20) associated with $\operatorname{det}(M)$ by geometric class field theory (cf. [6], Th. 5.25). Then we have an equality

$$
\left\langle\chi_{\operatorname{det}(M)}\right\rangle(\tilde{\pi})=\operatorname{det}\left(\operatorname{CFT}_{\tilde{\pi}}(M)\right)
$$

of characters of $G_{s}$, with the notation from ([6], Par. 7.11).

Indeed, we have

$$
\operatorname{det}\left(\operatorname{CFT}_{\tilde{\pi}}(M)\right)=\operatorname{CFT}_{\tilde{\pi}}(\operatorname{det}(M)) .
$$

By ([6], Par. 5.34), the pullback of the multiplicative local system $\chi_{\operatorname{det}(M)}$ by the morphism

$$
\mathbb{G}_{m, k} \rightarrow \operatorname{Pic}\left(S_{(s)}, D\right)_{s},
$$

which sends the coordinate $t$ to $1-t \pi^{-1}$, is the Gabber-Katz extension of $\operatorname{det}(M)$ with respect to the uniformiser $\pi$. We thus have

$$
\left(\chi_{\operatorname{det}(M)}\right)_{\mid 1-\pi^{-1}}=\operatorname{det}\left(\operatorname{CFT}_{\tilde{\pi}}(M)\right) ;
$$

hence the conclusion.

\section{9 .}

We still retain the notation from 4.5 . Let $\Lambda$ be a finite $\mathbb{Z} / \ell^{n} \mathbb{Z}$ algebra for some integer $n$. The profinite group $I_{\eta_{s}}$ admits a unique $p$-Sylow subgroup $P$, the wild inertia group associated with $\left(S_{(s)}, \overline{\eta_{s}}\right)$.

Definition 4.10. A continuous representation of $G_{\eta_{s}}$ on a $\Lambda$-module $M$ is totally wildly ramified if the submodule $M^{P}$ of $P$-invariant elements vanishes.

Example 4.11. Let $\psi: \mathbb{F}_{p} \rightarrow \Lambda^{\times}$be a nontrivial homomorphism and let $f$ be an element of $k\left(\eta_{s}\right)$ whose valuation is negative and prime to $p$. Then the continuous representation of $G_{\eta_{s}}$ corresponding to $\mathcal{L}_{\psi}\{f\}$ is totally wildly ramified. The latter assertion holds as well for $\mathcal{F} \otimes \mathcal{L}_{\psi}\{f\}$ for any tamely ramified lisse étale sheaf of $\Lambda$-modules $\mathcal{F}$ on $\eta_{s}$, because $P$ acts trivially on $\mathcal{F}_{\overline{\eta_{s}}}$. 
Proposition 4.12. Let $M$ be a $\Lambda$-module endowed with a continuous $\Lambda$-linear left action of $G_{\eta}$, and let $\mathcal{F}$ be the corresponding lisse étale sheaf of $\Lambda$-modules on $\eta$. If $M$ is totally wildly ramified (cf. 4.10) then the canonical homomorphism

$$
j_{!} \mathcal{F} \rightarrow R j_{*} \mathcal{F},
$$

where $j: \eta_{s} \rightarrow S_{(s)}$ is the canonical open immersion, is a quasi-isomorphism.

This amounts to the acyclicity of the fibre of $R j_{*} \mathcal{F}$ at $\bar{s}$; that is, to the vanishing of $H^{b}\left(I_{\eta_{s}}, M\right)$ for each integer $b$. We have the Hochschild-Serre spectral sequence

$$
H^{a}\left(I_{\eta_{s}} / P, H^{b}(P, M)\right) \Rightarrow H^{a+b}\left(I_{\eta_{s}}, M\right) .
$$

The groups $H^{b}(P, M)$ vanish for $b \neq 0$ because $P$ is a $p$-group and $M$ is of $\ell$-torsion, and $H^{0}(P, M)=M^{P}$ vanishes because $M$ is assumed to be totally wildly ramified. Thus, $H^{b}(P, M)$ vanishes for any $j$, and consequently so does $H^{b}\left(I_{\eta_{s}}, M\right)$.

Proposition 4.13. Let $M$ be a $\Lambda$-module endowed with a continuous $\Lambda$-linear left action of $I_{\eta_{s}}$. Then the canonical homomorphism

$$
R \Gamma_{c}\left(\mathbb{A}_{\bar{s}}^{1}, r_{\bar{s}, \pi}^{-1} M_{!} \otimes \mathcal{L}_{\psi}\{-t\}\right) \rightarrow R \Gamma\left(\mathbb{A}_{\bar{s}}^{1}, r_{\bar{s}, \pi}^{-1} M_{!} \otimes \mathcal{L}_{\psi}\{-t\}\right)
$$

is a quasi-isomorphism. Moreover, the complex $R \Gamma\left(\mathbb{A} \frac{1}{s}, r_{\bar{s}, \pi}^{-1} M_{!} \otimes \mathcal{L}_{\psi}\{-t\}\right)$ is concentrated in degree 1.

The first assertion follows from Example 4.11 and from Proposition 4.12. The second assertion follows from the vanishing of $H^{0}\left(\mathbb{A}_{\frac{1}{s}}, r_{\pi}^{-1} M_{!} \otimes \mathcal{L}_{\psi}\{-t\}\right)$ and from the fact that the étale cohomological dimension of a smooth affine curve over a separably closed field is at most 1 .

Corollary 4.14. The functor

$$
\operatorname{Art}_{\pi}: M \mapsto H_{c}^{1}\left(\mathbb{A}_{\bar{s}}^{1}, r_{\bar{s}, \pi}^{-1} M_{!} \otimes \mathcal{L}_{\psi}\{-t\}\right)
$$

from $I_{\eta_{s}}-\operatorname{Mod}_{\Lambda}$ to $\operatorname{Mod}_{\Lambda}$ is additive and exact and commutes with base change along homomorphisms $\Lambda \rightarrow \Lambda^{\prime}$ of finite $\mathbb{Z} / \ell^{n} \mathbb{Z}$ algebra.

This is an immediate consequence of Proposition 4.13. We note that $\mathrm{Art}_{\pi}$ is naturally endowed with a structure of $\Lambda$-linear $\left(G_{\eta_{s}}, I_{\eta_{s}}\right)$-contraction, with $(\tau(g))_{g \in G_{s}}$ constructed as in 4.5 .

Proposition 4.15. Let $\Lambda$ be a finite field of characteristic $\ell$. Let $M$ be a $\Lambda$-module endowed with a continuous $\Lambda$-linear left action of $G_{\eta_{s}}$. Then we have

$$
\begin{aligned}
\operatorname{rk}\left(\operatorname{Art}_{\pi}(M)\right) & =\operatorname{rk}(M)+\operatorname{sw}(M)=a\left(S_{(s)}, M_{!}\right), \\
\operatorname{det}\left(\operatorname{Art}_{\pi}(M)\right) & =\varepsilon\left(S_{(s)}, M_{!}, d \pi\right),
\end{aligned}
$$

where $a\left(S_{(s)},-\right)$ is the Artin conductor (cf. [6], Par. 7.2) and $\varepsilon\left(S_{(s)},-\right)$ is the geometric local $\varepsilon$-factor from ([6], Def. 9.2).

The first assertion follows from the Grothendieck-Ogg-Shafarevich formula, and the second one matches the definition of geometric local $\varepsilon$-factors in ([6], Def. 9.2). 
Corollary 4.16. Let $\Lambda$ be a finite field of characteristic $\ell$, and let $\mathcal{F}$ be an object of $D_{c}^{b}\left(S_{(s)}, \Lambda\right)$. Then we have

$$
\begin{aligned}
\operatorname{rk}\left(\operatorname{Art}_{\pi} R \Phi_{\text {id }}^{s}(\mathcal{F})\right) & =a\left(S_{(s)}, \mathcal{F}\right), \\
\operatorname{det}\left(\operatorname{Art}_{\pi} R \Phi_{\text {id }}^{s}(\mathcal{F})\right) & =\varepsilon\left(S_{(s)}, \mathcal{F}, d \pi\right) .
\end{aligned}
$$

This follows from Propositions 4.15 and 2.16 and from the fact that $\operatorname{Art}_{\pi}(\Lambda) \cong \Lambda$.

\section{Proof of Theorem 1.2}

In this section, we prove Theorem 1.2. The preliminary paragraph 5.1 provides a more detailed conclusion in the case of a projective line. We then explain how to reduce to projective schemes by using Chow's lemma in 5.2. Theorem 1.2 is then reduced to the case of projective spaces in 5.3, and the latter case is handled by induction on the dimension in 5.4. Throughout this section, we denote by $\Lambda$ a finite extension of $\mathbb{F}_{\ell}$.

\section{1 .}

Let $S=\mathbb{P}_{k}^{1}$, and let $\mathcal{F}$ be an object of $D_{c}^{b}(S, \Lambda)$. We denote by $t$ the canonical coordinate on the open subset $\mathbb{A}_{k}^{1}$ of $S$. By the product formula ([6], Th. 1.9), we have, with notation as in ([6], Par. 3.26),

$$
\begin{aligned}
\varepsilon_{\bar{k}}(S, \mathcal{F})= & \chi_{\mathrm{cyc}}^{-\mathrm{rk}(\mathcal{F})} \varepsilon\left(S_{(\infty)}, \mathcal{F}_{\mid S_{(\infty)}}, d t\right) \prod_{s \in\left|\mathbb{A}_{k}^{1}\right|} \delta_{s / k}^{a\left(S_{(s)}, \mathcal{F}_{\mid S}(s)\right.} \operatorname{Ver}_{s / k}\left(\varepsilon\left(S_{(s)}, \mathcal{F}_{\mid S_{(s)}}, d t\right)\right) \\
= & \chi_{\mathrm{cyc}}^{\operatorname{rk}(\mathcal{F})}\left\langle\chi_{\operatorname{det}\left(\mathcal{F}_{\eta_{\infty}}\right)}\right\rangle\left(-t^{2}\right) \varepsilon\left(S_{(\infty)}, \mathcal{F}_{\mid S_{(\infty)}}, d\left(t^{-1}\right)\right) \\
& \prod_{s \in\left|\mathbb{A}_{k}^{1}\right|} \delta_{s / k}^{a\left(S_{(s)}, \mathcal{F}_{\mid S(s)}\right)} \operatorname{Ver}_{s / k}\left(\varepsilon\left(S_{(s)}, \mathcal{F}_{\left.\mid S_{(s)}\right)}, d t\right)\right) .
\end{aligned}
$$

For any closed point $s$ of $\mathbb{A}_{k}^{1}$, we have $d t=d(t-t(s))$ and $\pi_{s}=t-t(s)$ is a uniformiser of $S_{(s)}$. For $s=\infty$, we set $\pi_{\infty}=t^{-1}$. Then Corollary 4.16 yields

$$
\delta_{s / k}^{a\left(S_{(s)}, \mathcal{F}_{\mid S(s)}\right)} \operatorname{Ver}_{s / k}\left(\varepsilon\left(S_{(s)}, \mathcal{F}_{\mid S_{(s)}}, d \pi_{s}\right)\right)=\operatorname{det}\left(\operatorname{Ind}_{G_{s}}^{G_{k}} \operatorname{Art}_{\pi_{s}} R \Phi_{\mathrm{id}}^{s}(\mathcal{F})\right),
$$

for any closed point $s$ of $\mathbb{P}_{k}^{1}$. By Proposition 4.8, we further have

$$
\left\langle\chi_{\operatorname{det}\left(\mathcal{F}_{\eta_{\infty}}\right)}\right\rangle\left(-t^{2}\right)=\operatorname{det}\left(\mathrm{CFT}_{t^{-1}}\left(\mathcal{F}_{\eta_{\infty}}\right)\right)^{-1} \operatorname{det}\left(\mathrm{CFT}_{-t^{-1}}\left(\mathcal{F}_{\eta_{\infty}}\right)\right)^{-1} .
$$

We thus obtain that $\varepsilon_{\bar{k}}(S, \mathcal{F})$ coincides with

$$
\operatorname{det}\left(\mathrm{CFT}_{t^{-1}} R \Psi_{\mathrm{id}}^{\infty} \mathcal{F}(-1)\right)^{-1} \operatorname{det}\left(\mathrm{CFT}_{-t^{-1}} R \Psi_{\mathrm{id}}^{\infty} \mathcal{F}\right)^{-1} \prod_{s \in\left|\mathbb{P}_{k}^{1}\right|} \operatorname{det}\left(\operatorname{Ind}_{G_{s}}^{G_{k}} \operatorname{Art}_{\pi_{s}} R \Phi_{\mathrm{id}}^{s}(\mathcal{F})\right) .
$$

Moreover, the sum

$$
-\operatorname{rk}\left(\mathrm{CFT}_{t^{-1}} R \Psi_{\mathrm{id}}^{\infty} \mathcal{F}(-1)\right)-\operatorname{rk}\left(\mathrm{CFT}_{-t^{-1}} R \Psi_{\mathrm{id}}^{\infty} \mathcal{F}\right)+\sum_{s \in\left|\mathbb{P}_{k}^{1}\right|} \operatorname{rk}\left(\operatorname{Ind}_{G_{s}}^{G_{k}} \operatorname{Art}_{\pi_{s}} R \Phi_{\mathrm{id}}^{s}(\mathcal{F})\right)
$$


is equal, by Corollary 4.16, to

$$
-2 \operatorname{rk}(\mathcal{F})+\sum_{s \in\left|\mathbb{P}_{k}^{1}\right|}[k(s): k] a\left(S_{(s)}, \mathcal{F}_{S_{(s)}}\right) .
$$

The latter sum is equal to the Euler characteristic $-\chi(S, \mathcal{F})$ by the Grothendieck-OggShafarevich formula.

\section{2 .}

Let us assume that Theorem 1.2 is proved for any projective $k$-scheme. Let us then prove that Theorem 1.2 holds for any proper $k$-scheme $X$. We argue by induction on the dimension of $X$, which is henceforth assumed to be at least 1 because 0 -dimensional schemes are projective.

By Chow's lemma, there exists a proper morphism $f: X^{\prime} \rightarrow X$, which is an isomorphism above a dense open subset $U$ of $X$, such that $X^{\prime}$ is projective. Let $Z^{\prime}=X^{\prime} \backslash f^{-1}(U)$ and $Z=X \backslash U$. Because $X^{\prime}$ and $Z^{\prime}$ are projective, and because $Z$ has dimension strictly smaller than $X$, the preliminary assumption and the induction hypothesis yield that Theorem 1.2 holds for $X^{\prime}, Z^{\prime}$ and $Z$; hence the existence of collections $\left(E_{X^{\prime}, x^{\prime}}\right)_{x^{\prime} \in\left|X^{\prime}\right|},\left(E_{Z^{\prime}, z^{\prime}}\right)_{z^{\prime} \in\left|Z^{\prime}\right|}$ and $\left(E_{Z, z}\right)_{z \in|Z|}$ of triangulated functors satisfying the conclusion of Theorem 1.2 for $X^{\prime}, Z^{\prime}$ and $Z$, respectively.

Let $\mathcal{F}$ be an object of $D_{c}^{b}(X, \Lambda)$. Then $\varepsilon_{\bar{k}}(X, \mathcal{F})$ coincides with $\varepsilon_{\bar{k}}(Z, \mathcal{F}) \varepsilon_{\bar{k}}\left(X^{\prime}, \mathcal{F}\right) \varepsilon_{\bar{k}}$ $\left(Z^{\prime}, \mathcal{F}\right)^{-1}$ and is therefore equal to the determinant of

$$
\begin{aligned}
& \bigoplus_{z \in|Z|} \operatorname{Ind}_{G_{z}}^{G_{k}} E_{Z, z}\left(\mathcal{F}_{\mid Z_{(z)}}\right) \oplus \bigoplus_{x^{\prime} \in\left|X^{\prime}\right|} \operatorname{Ind}_{G_{x^{\prime}}}^{G_{k}} E_{X^{\prime}, x^{\prime}}\left(f^{-1} \mathcal{F}_{\mid X_{\left(x^{\prime}\right)}^{\prime}}\right) \oplus \\
& \bigoplus_{z^{\prime} \in\left|Z^{\prime}\right|} \operatorname{Ind}_{G_{z^{\prime}}}^{G_{k}} E_{Z^{\prime}, z^{\prime}}\left(f^{-1} \mathcal{F}_{\mid Z_{\left(z^{\prime}\right)}^{\prime}}\right)[1] .
\end{aligned}
$$

This yields the conclusion by setting for any closed point $x$ of $X$,

$$
E_{x}(\mathcal{G})=E_{Z, x}\left(\mathcal{G}_{\mid Z_{(x)}}\right) \oplus \bigoplus_{x^{\prime} \in\left|f^{-1}(x)\right|} \operatorname{Ind}_{G_{x^{\prime}}}^{G_{x}}\left(E_{X^{\prime}, x^{\prime}}\left(f^{-1} \mathcal{G}_{\mid X_{\left(x^{\prime}\right)}^{\prime}}\right) \oplus E_{Z^{\prime}, x^{\prime}}\left(f^{-1} \mathcal{G}_{\mid Z_{\left(x^{\prime}\right)}^{\prime}}\right)[1]\right)
$$

if $x$ belongs to $Z$ and

$$
E_{X, x}(\mathcal{G})=E_{X^{\prime}, f^{-1}(x)}\left(f^{-1} \mathcal{G}_{\left.\mid X_{(f-1}^{\prime}(x)\right)}\right)
$$

otherwise.

\section{3.}

Let $\iota: X \rightarrow \mathbb{P}_{k}^{d}$ be a closed immersion into a projective space $\mathbb{P}_{k}^{d}$, for some integer $d$. If Theorem 1.2 holds for $\mathbb{P}_{k}^{d}$, so that we have a collection $\left(E_{\mathbb{P}_{k}^{d}, x}\right)_{x \in\left|\mathbb{P}_{k}^{d}\right|}$ of triangulated functors, then Theorem 1.2 also holds for $X$ by setting

$$
E_{x}(\mathcal{G})=E_{\mathbb{P}_{k}^{d}, \iota(x)}\left(\iota_{*} \mathcal{G}\right) .
$$




\section{4 .}

Let us prove Theorem 1.2. By 5.2 and 5.3, it is sufficient to consider the case where $X=\mathbb{P}(V)$ is the projective space parametrising hyperplanes in a $k$-vector space $V$. We denote by $d+1$ the dimension of $V$, and we argue by induction on $d$, the case $d=0$ being immediate. We henceforth assume that $d$ is greater than 0 .

Let $e_{1}, e_{2}$ be a basis of a 2-dimensional sub- $k$-vector space $W$ of $V$ and let $X^{\prime}$ be the flag variety parametrising pairs $\left(\left[t_{1}: t_{2}\right], H\right)$, where $\left[t_{1}: t_{2}\right]$ is a point of $\mathbb{P}_{k}^{1}$ and $H$ is an hyperplane in $V$ containing $t_{1} e_{1}+t_{2} e_{2}$. Let $f: X^{\prime} \rightarrow \mathbb{P}_{k}^{1}$ and $r: X^{\prime} \rightarrow \mathbb{P}(V)$ be the first and second projections, respectively.

Let $\mathcal{F}$ be an object of $D_{c}^{b}(\mathbb{P}(V), \Lambda)$. The morphism $r$ is an isomorphism outside the closed subscheme $\iota: \mathbb{P}(V / W) \rightarrow \mathbb{P}(V)$ and is a $\mathbb{P}^{1}$-bundle above $\mathbb{P}(V / W)$; hence,

$$
\begin{aligned}
\varepsilon_{\bar{k}}(\mathbb{P}(V), \mathcal{F}) & =\varepsilon_{\bar{k}}\left(X^{\prime}, r^{-1} \mathcal{F}\right) \varepsilon_{\bar{k}}\left(\mathbb{P}(V / W), \iota^{-1} \mathcal{F}(-1)\right)^{-1} \\
& =\varepsilon_{\bar{k}}\left(\mathbb{P}_{k}^{1}, R f_{*} r^{-1} \mathcal{F}\right) \varepsilon_{\bar{k}}\left(\mathbb{P}(V / W), \iota^{-1} \mathcal{F}(-1)\right)^{-1} .
\end{aligned}
$$

By 5.1, the homomorphism $\varepsilon_{\bar{k}}\left(\mathbb{P}_{k}^{1}, R f_{*} r^{-1} \mathcal{F}\right)$ is the determinant of

$$
\mathrm{CFT}_{t^{-1}} R \Psi_{\mathrm{id}}^{\infty} \mathcal{G}[1](-1) \oplus \mathrm{CFT}_{-t^{-1}} R \Psi_{\mathrm{id}}^{\infty} \mathcal{G}[1] \oplus \bigoplus_{s \in\left|\mathbb{P}_{k}^{1}\right|} \operatorname{Ind}_{G_{s}}^{G_{k}} \operatorname{Art}_{\pi_{s}} R \Phi_{\mathrm{id}}^{s}(\mathcal{G}),
$$

where $\mathcal{G}=R f_{*} r^{-1} \mathcal{F}$. Moreover, by 3.8 we have a functorial isomorphism

$$
\operatorname{Art}_{\pi_{s}} R \Phi_{\mathrm{id}}^{s}(\mathcal{G}) \simeq R \Gamma\left(X_{\bar{s}}^{\prime}, \operatorname{Art}_{\pi_{s}} R \Phi_{f}^{s}\left(r^{-1} \mathcal{F}\right)\right) .
$$

We similarly have

$$
\begin{aligned}
\mathrm{CFT}_{t^{-1}} R \Psi_{\mathrm{id}}^{\infty} \mathcal{G}(-1) & \cong R \Gamma\left(X_{\bar{s}}^{\prime}, \mathrm{CFT}_{t^{-1}} R \Psi_{f}^{\infty}\left(r^{-1} \mathcal{F}(-1)\right)\right) \\
\mathrm{CFT}_{-t^{-1}} R \Psi_{\mathrm{id}}^{\infty} \mathcal{G} & \cong R \Gamma\left(X_{\bar{s}}^{\prime}, \mathrm{CFT}_{-t^{-1}} R \Psi_{f}^{\infty}\left(r^{-1} \mathcal{F}\right)\right) .
\end{aligned}
$$

For each closed point $s=\left[t_{1}, t_{2}\right]$ of $\mathbb{P}_{k}^{1}$, the fibre $X_{s}^{\prime}$ is the projective space associated with the quotient of $V \otimes_{k} k(s)$ by the $k(s)$-line $k(s)\left(t_{1} e_{1}+t_{2} e_{2}\right)$. In particular, the induction hypothesis applies to $X_{s}^{\prime}$, and we therefore have a collection $\left(E_{X_{s}^{\prime}, x^{\prime}}\right)_{x^{\prime} \in\left|X_{s}^{\prime}\right|}$ of triangulated functors satisfying the conclusion of Theorem 1.2 for the $s$-scheme $X_{s}^{\prime}$. Thus, $\varepsilon_{\bar{k}}\left(\mathbb{P}_{k}^{1}, R f_{*} r^{-1} \mathcal{F}\right)$ is the determinant of

$$
\begin{aligned}
& \bigoplus_{x^{\prime} \in\left|X_{\infty}^{\prime}\right|} \operatorname{Ind}_{G_{x^{\prime}}}^{G_{k}} E_{X_{\infty}^{\prime}, x^{\prime}}\left(\mathrm{CFT}_{t^{-1}} R \Psi_{f}^{\infty}\left(r^{-1} \mathcal{F}(-1)\right)\right) \\
\oplus & \bigoplus_{x^{\prime} \in\left|X_{\infty}^{\prime}\right|} \operatorname{Ind}_{G_{x^{\prime}}}^{G_{k}} E_{X_{\infty}^{\prime}, x^{\prime}}\left(\mathrm{CFT}_{-t^{-1}} R \Psi_{f}^{\infty}\left(r^{-1} \mathcal{F}\right)\right) \\
\oplus & \bigoplus_{x^{\prime} \in\left|X^{\prime}\right|} \operatorname{Ind}_{G_{x^{\prime}}}^{G_{k}} E_{X_{f\left(x^{\prime}\right)}^{\prime}, x^{\prime}}\left(\operatorname{Art}_{\pi_{f\left(x^{\prime}\right)}} R \Phi_{f}^{f\left(x^{\prime}\right)}\left(r^{-1} \mathcal{F}\right)\right) .
\end{aligned}
$$

One should note that $r^{-1} \mathcal{F}$ is universally locally acyclic with respect to $f$ above a dense open subscheme of $\mathbb{P}_{k}^{1}$ by ([4], [Th. finitude] Theorem 2.13); hence, $R \Phi_{f}^{s}\left(r^{-1} \mathcal{F}\right)$ vanishes for all but finitely many closed points $s$ of $\mathbb{P}_{k}^{1}$. This and the induction hypothesis ensure that only finitely many terms contribute in the above sum. The conclusion then follows from 5.4.2 and from the induction hypothesis applied to $\mathbb{P}(V / W)$ : The property $1.2(1)$ 
has already been discussed, the property 1.2(3) holds by construction and it remains to clarify why $1.2(2)$ holds as well. For a point $x^{\prime} \in\left|X_{\infty}^{\prime}\right|$ and for an object $\mathcal{G}$ of $D_{c}^{b}\left(r\left(x^{\prime}\right), \Lambda\right)$, the pullback by the specialisation map sp : $\left(\mathbb{P}(V)_{\left(r\left(x^{\prime}\right)\right)}\right)_{\text {é } t} \rightarrow r\left(x^{\prime}\right)_{\text {ét }}$ yields an object $\mathrm{sp}^{-1} \mathcal{G}$ of $D_{c}^{b}\left(\mathbb{P}(V)_{\left(r\left(x^{\prime}\right)\right)}, \Lambda\right)$. For $\mathcal{F}$ in $D_{c}^{b}\left(\mathbb{P}(V)_{\left(r\left(x^{\prime}\right)\right)}, \Lambda\right)$, we then have

$$
R \Psi_{f}^{\infty}\left(r^{-1}\left(\mathcal{F} \otimes \mathrm{sp}^{-1} \mathcal{G}\right)(-1)\right) \simeq R \Psi_{f}^{\infty}\left(r^{-1}(\mathcal{F}(-1))\right) \otimes r^{-1} \mathrm{sp}^{-1} \mathcal{G}_{\mid X_{\infty}^{\prime}} .
$$

Then 2.16 yields a functorial isomorphism

$$
\mathrm{CFT}_{t^{-1}} R \Psi_{f}^{\infty}\left(r^{-1}\left(\mathcal{F} \otimes \mathrm{sp}^{-1} \mathcal{G}\right)(-1)\right) \simeq \mathrm{CFT}_{t^{-1}} R \Psi_{f}^{\infty}\left(r^{-1} \mathcal{F}(-1)\right) \otimes r^{-1} \mathrm{sp}^{-1} \mathcal{G}_{\mid X_{\infty}^{\prime}} .
$$

Because $E_{X_{\infty}^{\prime}, x^{\prime}}$ satisfies the twist formula as well by induction, we obtain the property $1.2(2)$ for $E_{X_{\infty}^{\prime}, x^{\prime}}\left(\mathrm{CFT}_{t^{-1}} R \Psi_{f}^{\infty}\left(r^{-1} \mathcal{F}(-1)\right)\right)$. The same argument provides 1.2(2) for the other terms in 5.4.5; hence the conclusion.

\section{Proof of Theorem 1.8}

In this section, we prove Theorem 6.1 , of which Theorem 1.8 is an immediate consequence.

Theorem 6.1. Let $\Lambda$ be a finite field of characteristic $\ell$. Let $S$ be a Henselian trait of equicharacteristic $p$, with closed point $s$ such that $k(s)$ is perfect, and let $\omega$ be a nonzero meromorphic differential 1-form on $S$. Let $f: X \rightarrow S$ be a proper morphism. Then there exists a collection

$$
\left(E_{x}\right)_{x \in\left|X_{s}\right|}
$$

of triangulated functors $E_{x}: D_{c}^{b}\left(X_{(x)}, \Lambda\right) \rightarrow D_{c}^{b}(x, \Lambda)$, indexed by the set of closed points of the special fibre $X_{s}$, such that

(1) for any object $\mathcal{F}$ of $D_{c}^{b}(X, \Lambda)$, we have $E_{x}\left(\mathcal{F}_{\mid X_{(x)}}\right) \simeq 0$ for all but finitely many closed points $x$ of $X$;

(2) for any closed point $x$ of $X$, any object $\mathcal{F}$ of $D_{c}^{b}\left(X_{(x)}, \Lambda\right)$ and any object $\mathcal{G}$ of $D_{c}^{b}(x, \Lambda)$, we have an isomorphism

$$
E_{x}\left(\mathcal{F} \otimes \mathrm{sp}^{-1} \mathcal{G}\right) \simeq E_{x}(\mathcal{F}) \otimes \mathcal{G},
$$

where $\mathrm{sp}: X_{(x)} \rightarrow x$ is the canonical specialisation morphism, and this isomorphism is functorial in $\mathcal{F}$ and $\mathcal{G}$;

(3) for any object $\mathcal{F}$ of $D_{c}^{b}(X, \Lambda)$, we have

$$
\begin{aligned}
& \varepsilon_{\bar{s}}\left(S, R f_{*} \mathcal{F}, \omega\right)=\operatorname{det}\left(\bigoplus_{x \in\left|X_{s}\right|} \operatorname{Ind}_{G_{x}}^{G_{s}} E_{x}\left(\mathcal{F}_{\mid X_{(x)}}\right)\right), \\
& a\left(S, R f_{*} \mathcal{F}, \omega\right)=\operatorname{rk}\left(\bigoplus_{x \in\left|X_{s}\right|} \operatorname{Ind}_{G_{x}}^{G_{s}} E_{x}\left(\mathcal{F}_{\mid X_{(x)}}\right)\right),
\end{aligned}
$$

where $\bar{s}$ is a separable closure of $s$ with Galois group $G_{s}$ and where $a(S,-, \omega)$ is the Artin conductor (cf. [6], Par. 7.2). 
Let $\pi$ be a uniformiser of $S$, and let us write $\omega=\alpha d \pi$ for some nonzero element $\alpha$ of the function fields $k(\eta)$ of $S$. We denote by $v(\alpha)$ the valuation of $\alpha$ in the discretely valued field $k(\eta)$. For any object $\mathcal{G}$ of $D_{c}^{b}(S, \Lambda)$, we have

$$
\begin{aligned}
\varepsilon_{\bar{s}}(S, \mathcal{G}, \omega) & =\varepsilon_{\bar{s}}(S, \mathcal{G}, d \pi)\left\langle\chi_{\operatorname{det}\left(\mathcal{G}_{\eta}\right)}\right\rangle(\alpha) \chi_{\operatorname{cyc}}^{-v(\alpha) \operatorname{rk}\left(\mathcal{F}_{\eta}\right),} \\
a(S, \mathcal{G}, \omega) & =a(S, \mathcal{G})+v(\alpha) \operatorname{rk}\left(\mathcal{G}_{\eta}\right),
\end{aligned}
$$

with notation as in ([6], Def. 9.2) or Proposition 4.8. It is therefore enough to produce families $\left(E_{x}^{1}\right)_{x \in\left|X_{s}\right|}$ and $\left(E_{x}^{2}\right)_{x \in\left|X_{s}\right|}$ of triangulated functors as in Theorem 6.1 (1), (2) such that

$$
\begin{aligned}
\varepsilon_{\bar{s}}\left(S, R f_{*} \mathcal{F}, d \pi\right) & =\operatorname{det}\left(\bigoplus_{x \in\left|X_{s}\right|} \operatorname{Ind}_{G_{x}}^{G_{s}} E_{x}^{1}\left(\mathcal{F}_{\mid X_{(x)}}\right)\right), \\
a\left(S, R f_{*} \mathcal{F}\right) & =\operatorname{rk}\left(\bigoplus_{x \in\left|X_{s}\right|} \operatorname{Ind}_{G_{x}}^{G_{s}} E_{x}^{1}\left(\mathcal{F}_{\mid X_{(x)}}\right)\right),
\end{aligned}
$$

and

$$
\begin{aligned}
\left\langle\chi_{\operatorname{det}\left(\mathcal{G}_{\eta}\right)}\right\rangle(\alpha) \chi_{\mathrm{cyc}}^{-v(\alpha) \operatorname{rk}\left(\mathcal{F}_{\eta}\right)} & =\operatorname{det}\left(\bigoplus_{x \in\left|X_{s}\right|} \operatorname{Ind}_{G_{x}}^{G_{s}} E_{x}^{2}\left(\mathcal{F}_{\mid X_{(x)}}\right)\right) \\
v(\alpha) \operatorname{rk}\left(\mathcal{G}_{\eta}\right) & =\operatorname{rk}\left(\bigoplus_{x \in\left|X_{s}\right|} \operatorname{Ind}_{G_{x}}^{G_{s}} E_{x}^{2}\left(\mathcal{F}_{\mid X_{(x)}}\right)\right)
\end{aligned}
$$

In order to construct $\left(E_{x}^{1}\right)_{x \in\left|X_{s}\right|}$, we first notice that Corollary 4.16 and Proposition 3.8 yield that $\varepsilon_{\bar{s}}\left(S, R f_{*} \mathcal{F}, d \pi\right)$ and $a\left(S, R f_{*} \mathcal{F}\right)$ are respectively the determinant and rank of the complex

$$
\operatorname{Art}_{\pi} R \Phi_{\text {id }}^{s}\left(R f_{*} \mathcal{F}\right) \cong R \Gamma\left(X_{\bar{s}}, \operatorname{Art}_{\pi} R \Phi_{f}^{s}(\mathcal{F})\right) .
$$

If $\left(E_{X_{s}, x}\right)_{x \in\left|X_{s}\right|}$ is as in Theorem 1.2 applied to the proper $s$-scheme $X_{s}$, then we can set

$$
E_{x}^{1}(\mathcal{F})=E_{X_{s}, x}\left(\operatorname{Art}_{\pi} R \Phi_{f}^{s}(\mathcal{F})\right) .
$$

For $\left(E_{x}^{2}\right)_{x \in\left|X_{s}\right|}$, we can assume by additivity that $\alpha$ is a uniformiser on $S$, in which case Proposition 4.8 ensures that it is enough to set

$$
E_{x}^{2}(\mathcal{F})=E_{X_{s}, x}\left(\mathrm{CFT}_{\alpha} R \Psi_{f}^{s}(\mathcal{F})(-1)\right) .
$$

Remark 6.2. Let $X$ be a smooth projective curve of a field $k$ of positive characteristic $p$ and let $\mathcal{F}$ be an object of $D_{c}^{b}(X, \Lambda)$. The product formula for curves expresses the determinant of $R \Gamma\left(X_{\bar{k}}, \mathcal{F}\right)$ as a product of finitely many local $\varepsilon$-factors, which are invariants attached to Galois representations of 1-dimensional local fields. If $k$ is itself a 1-dimensional local field (i.e., a complete discrete valuation field with perfect residue field of positive characteristic) of positive characteristic, then the method described in this section decomposes the local $\varepsilon$-factor of $R \Gamma\left(X_{\bar{k}}, \mathcal{F}\right)$ as a product of finitely many contributions, namely, 2-dimensional local $\varepsilon$-factors, which are invariants attached to 
Galois representations of 2-dimensional local fields of positive characteristic (i.e., complete discrete valuation fields of positive characteristic whose residue fields are 1-dimensional local fields). By iterating this procedure, one can attach an $n$-dimensional local $\varepsilon$-factor to Galois representations of $n$-dimensional local fields of positive characteristic such that if $k$ is an $n$-dimensional local field of positive characteristic then the $n$-dimensional local $\varepsilon$-factor of $R \Gamma\left(X_{\bar{k}}, \mathcal{F}\right)$ factors as the product of finitely many $(n+1)$-dimensional local $\varepsilon$-factors.

Acknowledgements This work was prepared at the Institut des Hautes Études Scientifiques and the École Normale Supérieure while the author benefitted from their hospitality and support. This text is a continuation of the part [6] of the author's doctoral thesis, realised under the supervision of Ahmed Abbes. The author thanks Ahmed Abbes, Lei Fu, Ofer Gabber, Dennis Gaitsgory, Adriano Marmora, Deepam Patel, Takeshi Saito, Daichi Takeuchi and Enlin Yang for useful discussions. Further thanks go to the anonymous referees for their remarks and comments.

\section{References}

[1] P. Deligne, Les constantes des équations fonctionnelles des fonctions $L$ [Constants of functional equations of $L$-functions], in Modular Functions of One Variable II, Lecture Notes in Mathematics, Vol. 349, pp. 501-597 (Springer-Verlag, Berlin, 1973).

[2] P. Deligne, Séminaire de Géométrie Algébrique du Bois Marie-Cohomologie étale [Étale cohomology] (SGA 4 1/2), Lecture Notes in Mathematics, Vol. 569 (Springer-Verlag, Berlin, 1977).

[3] P. Deligne and N. Katz, Séminaire de Géométrie Algébrique du Bois Marie-1967-69Groupes de monodromie en géométrie algébrique [Monodromy groups in algebraic geometry] (SGA 7), Vol. 2, Lecture Notes in Mathematics, Vol. 340 (Springer-Verlag, Berlin, 1973).

[4] B. Dwork, On the Artin root number, Amer. J. Math. 78 (1956), 444-472.

[5] A. Grothendieck, Séminaire de Géométrie Algébrique du Bois Marie-1963-64-Théorie des topos et cohomologie étale des schémas [Topos theory and étale cohomology of schemes] $S G A$ 4), Lecture Notes in Mathematics, Vols. 269, 270, 305 (Springer-Verlag, Berlin, 1972-1973).

[6] Q. Guignard, Geometric local epsilon factors, arxiv:1902.06523 (v3), 2019.

[7] L. Illusie, Théorie de Brauer et caractéristique d'Euler-Poincaré d'après P. Deligne [Brauer theory and Euler-Poincaré characteristic, after P. Deligne], Astérisque 82-83 (1981), 161-172.

[8] K. Kato and S. Saito, Unramified class field theory of arithmetical surfaces, Ann. Math. 118 (1983), 241-275.

[9] N. M. Katz, Local-to-global extensions of representations of fundamental groups, Ann. Inst. Fourier 36(4) (1986), 69-106.

[10] G. Laumon, Transformation de Fourier, constantes d'équations fonctionnelles et conjecture de Weil [Fourier transform, constants of functional equations and Weil conjectures], Pub. Math. I.H.E.S. 65 (1987), 131-210.

[11] T. Saito, The characteristic cycle and the singular support of a constructible sheaf, Invent. Math. 207(2) (2017), 597-695.

[12] The Stacks Project Authors, Stacks Project, http://stacks.math.columbia.edu, 2019. 
[13] D. TAKeuchi, Characteristic epsilon cycles of $\ell$-adic sheaves on varieties, arxiv:1911.02269, 2019.

[14] N. Umezaki, E. YANG And Y. ZhaO, Characteristic class and the epsilon factor of an étale sheaf, arxiv:1701.02841, 2018.

[15] I. Vidal, Théorie de Brauer et conducteur de Swan [Brauer theory and Swan conductor], J. Algebr. Geom. 13 (2004), 349-391.

[16] S. YAsudA, Local $\varepsilon_{0}$-characters in torsion rings, J. Théor. Nombres Bordeaux 19 (2007), 763-797.

[17] S. Yasuda, Local constants in torsion rings, J. Math. Sci. (Univ. Tokyo) 16(2) (2009), 125-197.

[18] S. YASUdA, The product formula for local constants in torsion rings, J. Math. Sci. (Univ. Tokyo) 16(2) (2009), 199-230. 